\title{
A LOCAL REGULARIZATION OPERATOR FOR TRIANGULAR AND QUADRILATERAL FINITE ELEMENTS*
}

\author{
C. BERNARDI ${ }^{\dagger}$ AND V. GIRAULT ${ }^{\dagger}$
}

\begin{abstract}
This paper develops a local regularization operator on triangular or quadrilateral finite elements built on structured or unstructured meshes. This operator is a variant of the regularization operator of Clément; however, ours is constructed via a local projection in a reference domain. We prove in this paper that it has the same optimal approximation properties as the standard interpolation operator, and we present some applications.
\end{abstract}

Key words. regularization operator, triangular finite elements, quadrilateral finite elements

AMS subject classifications. Primary, 65D05; Secondary, 65N30

PII. S0036142995293766

Introduction. Let $\Omega$ be a two-dimensional bounded open set with a polygonal boundary $\Gamma$. Let $\mathcal{T}_{h}$ be a triangulation or quadrangulation of $\bar{\Omega}$, and let $\Theta_{h}$ be a standard associated finite element space. The purpose of this paper is to construct an operator $R_{h}$ that associates, with any function $u$ in $L^{1}(\Omega)$, an element $R_{h}(u)$ in $\Theta_{h}$ and satisfies the same local approximation properties as the usual interpolation operator when $u$ is sufficiently smooth. Since this operator must also act on functions that are not necessarily continuous, it replaces the nodal values of the function that is interpolated by adequate averages.

For triangular meshes, such operators were introduced by Clément in [7] and generalized by Bernardi in [2]. However, in contrast to [7], the averages in the present paper are computed in some reference domain; this idea was used in [2] to treat curved (isoparametric) triangles or simplices and also allows for an extension to quadrilateral meshes. In contrast to [2], they are computed on spaces of piecewise polynomial functions. Indeed, we will show by a simple counterexample that this is necessary to recover the usual interpolation error when the function that must be approximated is smooth.

Several modified versions of these operators exist; see [4, Chap. 4]. For instance, Scott and Zhang [14] use averages on the boundary of the elements, in particular when the associated degrees of freedom are on the boundary. The advantages are that, on one hand, the corresponding operator preserves the nullity of traces and that, on the other hand, it leaves invariant the functions of the discrete space. However, the drawback is that it is only defined on more regular functions, i.e., sufficiently smooth to have a trace on the boundary of elements. For this reason, we prefer first to construct a general operator and second to modify it in order that the new operator preserves the nullity of traces.

This paper is organized as follows. In section 1, we make precise the notation and we recall some basic results. We have chosen to treat separately, in sections 2 and 3 , the discussion of the averaging process on triangular finite elements and on

\footnotetext{
* Received by the editors October 26, 1995; accepted for publication (in revised form) June 25, 1997; published electronically August 25, 1998.

http://www.siam.org/journals/sinum/35-5/29376.html

${ }^{\dagger}$ Laboratoire d'Analyse Numérique, C.N.R.S. et Université Pierre et Marie Curie, B.C. 187, 4 Place Jussieu, 75252 Paris cedex 05, France (bernardi@ann.jussieu.fr, girault@ann.jussieu.fr). Part of the work of the second author was done while visiting the Indian Institute of Science in Bangalore (India) and was supported by the CEFIPRA Contract IFC/501-1/93/1553.
} 
quadrilateral finite elements, because the techniques involved are somewhat different, especially in the case of non-Cartesian quadrilateral meshes. In section 4, the error estimates for these averages are used to derive the error estimates for the corresponding regularization operator. Section 5 is devoted to some applications.

1. Preliminaries and notation. Let $\mathcal{O}$ be a bounded domain in $\mathbb{R}^{2}$ with a Lipschitz-continuous boundary $\partial \mathcal{O}$. We denote by $|\mathcal{O}|$ the measure of $\mathcal{O}$. For any nonnegative integer $m$ and any number $p$ with $1 \leq p \leq \infty$, we use the standard Sobolev spaces

$$
W^{m, p}(\mathcal{O})=\left\{v \in L^{p}(\mathcal{O}) ; \frac{\partial^{k} v}{\partial x_{1}^{i} \partial x_{2}^{k-i}} \in L^{p}(\mathcal{O}), 0 \leq i \leq k, 1 \leq k \leq m\right\},
$$

equipped with the two seminorms

$$
\begin{gathered}
|v|_{W^{m, p}(\mathcal{O})}=\left(\sum_{i=0}^{m}\left\|\frac{\partial^{m} v}{\partial x_{1}^{i} \partial x_{2}^{m-i}}\right\|_{L^{p}(\mathcal{O})}^{p}\right)^{1 / p}, \\
{[v]_{W^{m, p}(\mathcal{O})}=\left(\left\|\frac{\partial^{m} v}{\partial x_{1}^{m}}\right\|_{L^{p}(\mathcal{O})}^{p}+\left\|\frac{\partial^{m} v}{\partial x_{2}^{m}}\right\|_{L^{p}(\mathcal{O})}^{p}\right)^{1 / p}}
\end{gathered}
$$

and norm

$$
\|v\|_{W^{m, p}(\mathcal{O})}=\left(\sum_{k=0}^{m}|v|_{W^{k, p}(\mathcal{O})}^{p}\right)^{1 / p}
$$

with the usual modification for $p=\infty$. By interpolation, this definition can be extended to nonintegral values of $m$. In particular, for $1 \leq p<\infty$, fractional order spaces include the trace space of functions of $W^{1, p}(\mathcal{O})$, that is, $W^{1-1 / p, p}(\partial \mathcal{O})$, equipped with the norm

$$
\|\mu\|_{W^{1-1 / p, p}(\partial \mathcal{O})}=\inf _{v \in W^{1, p}(\mathcal{O}), v_{\mid \partial \mathcal{O}}=\mu}\|v\|_{W^{1, p}(\mathcal{O})} .
$$

The reader is referred to Lions and Magenes [12, Chap. 1] for fractional-order Sobolev spaces.

Finally, let us recall two fundamental results of polynomial interpolation. For any nonnegative integer $k$, let $\mathbb{P}_{k}$ be the space of polynomials in two variables of total degree less than or equal to $k$, and let $\mathbb{Q}_{k}$ be the space of polynomials in two variables of degree less than or equal to $k$ in each variable. Note that $\mathbb{P}_{k}$ and $\mathbb{Q}_{k}$ coincide for $k=0$, but otherwise $\mathbb{Q}_{k}$ is a subspace of $\mathbb{P}_{2 k}$. For any nonnegative integers $k$ and $\ell$, the polynomial spaces $\mathbb{P}_{k}$ and $\mathbb{Q}_{k}$ are contained in $W^{\ell, p}(\mathcal{O})$, and we can define the quotient spaces $W^{\ell, p}(\mathcal{O}) / \mathbb{P}_{k}$ and $W^{\ell, p}(\mathcal{O}) / \mathbb{Q}_{k}$, which are also Banach spaces equipped with the quotient norms

$$
\begin{aligned}
& \forall \dot{v} \in W^{\ell, p}(\mathcal{O}) / \mathbb{P}_{k},\|\dot{v}\|_{W^{\ell, p}(\mathcal{O}) / \mathbb{P}_{k}}=\inf _{r \in \mathbb{P}_{k}}\|v+r\|_{W^{\ell, p}(\mathcal{O})}, \\
& \forall \dot{v} \in W^{\ell, p}(\mathcal{O}) / \mathbb{Q}_{k},\|\dot{v}\|_{W^{\ell, p}(\mathcal{O}) / \mathbb{Q}_{k}}=\inf _{r \in \mathbb{Q}_{k}}\|v+r\|_{W^{\ell, p}(\mathcal{O})} .
\end{aligned}
$$


The next two theorems state important properties of these quotient spaces. The first one is proven in Deny and Lions [8] (cf. also Nečas [13, Chap. 1]) and the second one in Ciarlet and Raviart [6]. A more general result, in the finite union of starshaped domains with respect to balls, is proven by Dupont and Scott [9] and also by Durán [10] in a constructive way. This construction, inspired by Sobolev's explicit representation of a function as a polynomial plus a remainder term, is based on the representation of a function as an averaged Taylor's series. We refer to [4] for more details.

TheOREM 1.1. Assume that $\mathcal{O}$ is a bounded and connected open set in $\mathbb{R}^{2}$ with a Lipschitz-continuous boundary. For each integer $k \geq 0$ and number $p$ with $1 \leq p \leq \infty$, there exists a constant $C$ such that

$$
\forall v \in W^{k+1, p}(\mathcal{O}),\|\dot{v}\|_{W^{k+1, p}(\mathcal{O}) / \mathbb{P}_{k}} \leq C|v|_{W^{k+1, p}(\mathcal{O})} .
$$

TheOREM 1.2. Assume that $\mathcal{O}$ is a bounded and connected open set in $\mathbb{R}^{2}$ with a Lipschitz-continuous boundary. For each integer $k \geq 0$ and number $p$, with $1 \leq p \leq \infty$, there exists a constant $C$ such that

$$
\forall v \in W^{k+1, p}(\mathcal{O}),\|\dot{v}\|_{W^{k+1, p}(\mathcal{O}) / \mathbb{Q}_{k}} \leq C[v]_{W^{k+1, p}(\mathcal{O})} .
$$

2. A projection operator on triangular meshes. Let $h$ be a positive discretization parameter. Recall (cf. Ciarlet [5, Chap. II]) that a triangulation $\mathcal{T}_{h}$ of $\bar{\Omega}$ is a partition of $\bar{\Omega}$ into nondegenerate triangles $T$ with diameter bounded by $h$, such that each pair of triangles $T_{1}$ and $T_{2}$ of $\mathcal{T}_{h}$ are either disjoint or share a vertex or a complete side. We denote by $h_{T}$ the diameter of $T$, by $\rho_{T}$ the diameter of the circle inscribed in $T$, and we set

$$
\sigma_{T}=\frac{h_{T}}{\rho_{T}}
$$

We assume that the family of triangulations $\left(\mathcal{T}_{h}\right)_{h}$ is regular, i.e., there exists a constant $\sigma$, independent of $h$, such that

$$
\forall T \in \mathcal{T}_{h}, \sigma_{T} \leq \sigma .
$$

Let us fix a positive integer $k$, and let $\Theta_{h}$ be the standard finite element space

$$
\Theta_{h}=\left\{\theta_{h} \in \mathcal{C}^{0}(\bar{\Omega}) ; \forall T \in \mathcal{T}_{h}, \theta_{h \mid T} \in \mathbb{P}_{k}\right\}
$$

This definition must be completed by specifying the degrees of freedom of the functions of $\Theta_{h}$ : for the sake of simplicity, we assume that, in each triangle $T$, the degrees of freedom of a function $\theta_{h}$ in $\Theta_{h}$ are the values of $\theta_{h}$ on the principal lattice of order $k$, as in the example of Figure 1. In other words, the degrees of freedom of $\theta_{h}$ are its values at a set of particular nodes of the triangulation $\mathcal{T}_{h}$. Let $N$ be the number of these nodes and let $\left\{\boldsymbol{a}_{i}, 1 \leq i \leq N\right\}$ denote this set of nodes. For any node $\boldsymbol{a}_{i}$, let the macroelement $\Delta_{i}$ be the union of the triangles of $\mathcal{T}_{h}$ that share this node $\boldsymbol{a}_{i}$, as in Figure 2.

Remark 1. The results below still hold for more general degrees of freedom defined by linear functionals, if these functionals are continuous on functions in $\mathcal{C}^{0}(\bar{\Omega})$. But our proofs are not valid for Hermite-type finite elements, for instance. 


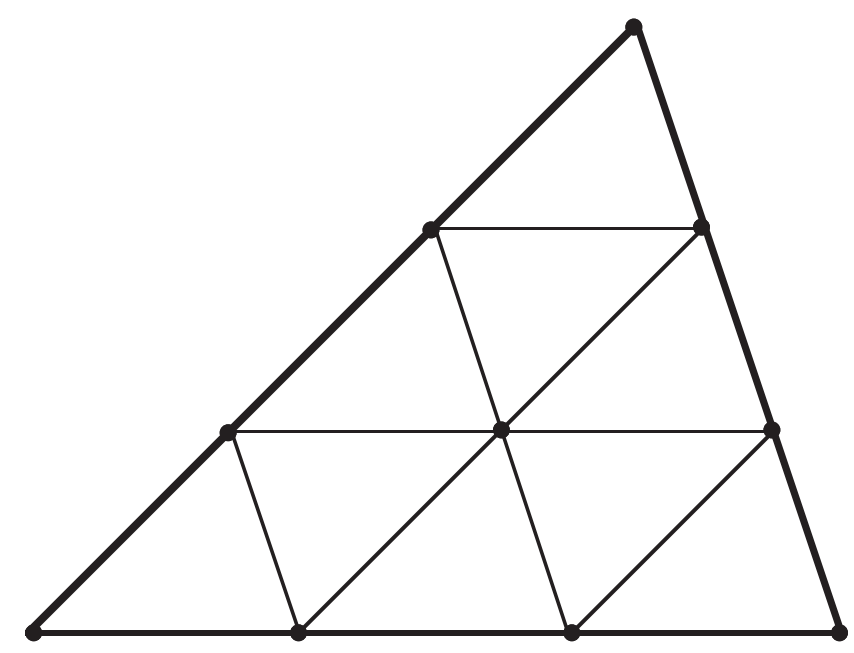

FIG. 1

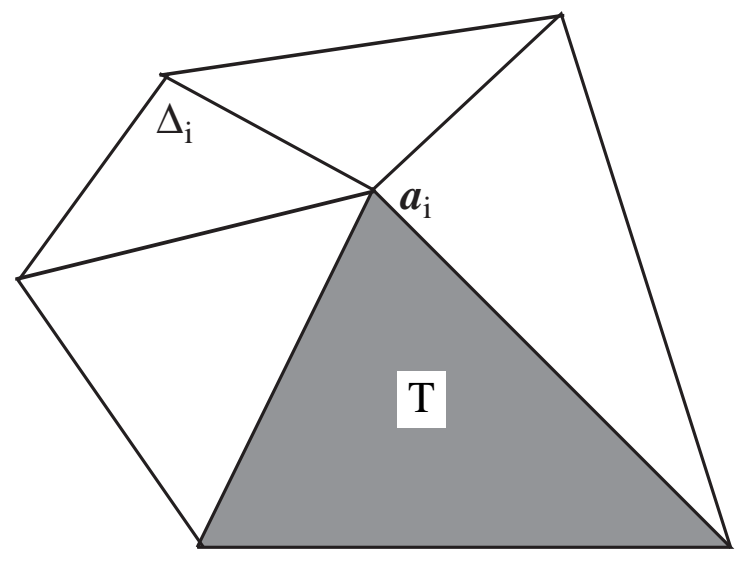

FIG. 2

We set

$$
h_{\Delta_{i}}=\sup _{T \subset \Delta_{i}} h_{T}, \quad \rho_{\Delta_{i}}=\inf _{T \subset \Delta_{i}} \rho_{T}, \quad \sigma_{\Delta_{i}}=\frac{h_{\Delta_{i}}}{\rho_{\Delta_{i}}} .
$$

Since the family of triangulations $\left(\mathcal{T}_{h}\right)_{h}$ is regular, it can be proved that (cf. Bernardi [2], Clément [7])

(i) there exists a constant $L$, independent of $h$, such that, for $1 \leq i \leq N, \Delta_{i}$ consists of at most $L$ triangles $T$ (more precisely, if $\boldsymbol{a}_{i}$ lies in the interior of $T$, then $\Delta_{i}$ coincides with $T$; if $\boldsymbol{a}_{i}$ lies on one side of $T$, then $\Delta_{i}$ consists of either two triangles, or only one if that side is a part of $\Gamma$, and if $\boldsymbol{a}_{i}$ is a vertex of $T$, then $\Delta_{i}$ has at most $L$ triangles);

(ii) there exists a constant $\hat{c}_{1}$, independent of $h$, such that, for $1 \leq i \leq N$,

$$
\forall T \subset \Delta_{i}, h_{\Delta_{i}} \leq \hat{c}_{1} h_{T} ;
$$

(iii) there exists a constant $\hat{c}_{2}$, independent of $h$, such that, for $1 \leq i \leq N$,

$$
\sigma_{\Delta_{i}} \leq \hat{c}_{2} \sigma .
$$



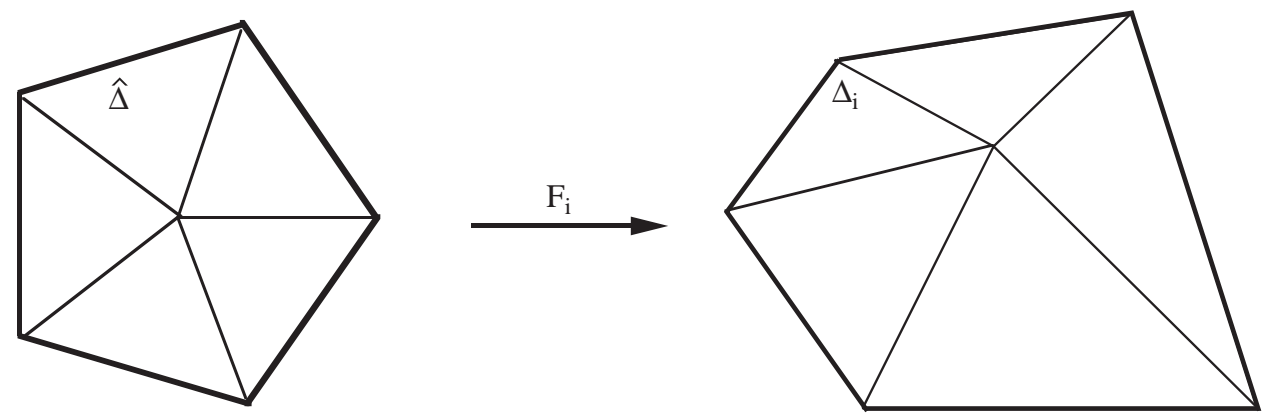

FIG. 3

Note also that

(iv) there exists a constant $K$, independent of $h$ (in fact, $K=(k+1)(k+2) / 2$ ), such that any $T$ in $\mathcal{T}_{h}$ belongs to at most $K$ macroelements $\Delta_{i}$.

Consider a macroelement $\Delta_{i}$ made of, say, $J$ triangles; we associate with $\Delta_{i}$ a reference macroelement $\hat{\Delta}_{i}$, made of $J$ equal isosceles reference unit triangles $\hat{T}_{j}$, as in Figure 3 . Owing to property (i), there exists only a fixed number $\hat{L}$ of different reference macroelements $\hat{\Delta}_{i}$, for $1 \leq i \leq N$, where $\hat{L}$ is independent of $h$. Therefore, since all the geometric characteristics of these reference macroelements can be bounded by constants independent of $h$, to alleviate notation we shall not distinguish them and suppress their index $i$, thus denoting them indifferently by $\hat{\Delta}$. It can be easily proved that, for each macroelement $\Delta_{i}$, there exists a continuous and invertible mapping $F_{i}$ that is affine on each reference triangle $\hat{T}$ of $\hat{\Delta}$ :

$$
\forall \hat{\boldsymbol{x}} \in \hat{T}, F_{i}(\hat{\boldsymbol{x}})=B_{T} \hat{\boldsymbol{x}}+\boldsymbol{b}_{T},
$$

such that

$$
\Delta_{i}=F_{i}(\hat{\Delta})
$$

It follows from the above construction that each matrix $B_{T}$ is nonsingular and

$$
\left\|B_{T}\right\| \leq \hat{c}_{3} h_{T}, \quad\left\|B_{T}^{-1}\right\| \leq \frac{\hat{c}_{4}}{\rho_{T}}, \quad \hat{c}_{5} \rho_{T}^{2} \leq\left|\operatorname{det}\left(B_{T}\right)\right| \leq \hat{c}_{6} h_{T}^{2} .
$$

We associate with $\Theta_{h}$ the local finite element spaces

$$
\begin{gathered}
\Theta(\hat{\Delta})=\left\{\hat{\theta} \in \mathcal{C}^{0}(\hat{\Delta}) ; \forall \hat{T} \subset \hat{\Delta}, \hat{\theta}_{\mid \hat{T}} \in \mathbb{P}_{k}\right\}, \\
\Theta\left(\Delta_{i}\right)=\left\{\theta \in \mathcal{C}^{0}\left(\Delta_{i}\right) ; \forall T \subset \Delta_{i}, \theta_{\mid T} \in \mathbb{P}_{k}\right\} .
\end{gathered}
$$

Then, for any function $\hat{u}$ in $L^{1}(\hat{\Delta})$, we define $\hat{r}(\hat{u})$ in $\Theta(\hat{\Delta})$ by

$$
\forall \hat{\theta} \in \Theta(\hat{\Delta}), \int_{\hat{\Delta}}(\hat{r}(\hat{u})-\hat{u}) \hat{\theta} d \hat{\boldsymbol{x}}=0,
$$

and, for any function $u$ in $L^{1}\left(\Delta_{i}\right)$, we define $r_{i}(u)$ in $\Theta\left(\Delta_{i}\right)$ by

$$
r_{i}(u) \circ F_{i}=\hat{r}\left(u \circ F_{i}\right),
$$


a relation that is often denoted symbolically in the literature by

$$
\widehat{r_{i}(u)}=\hat{r}(\hat{u}) \text {. }
$$

Clearly, $\hat{r}$ is a projection operator from $L^{1}(\hat{\Delta})$ onto $\Theta(\hat{\Delta})$ (orthogonal in $L^{2}(\hat{\Delta})$ ). But in general, the operator $r_{i}$, which is continuous from $L^{1}\left(\Delta_{i}\right)$ onto $\Theta\left(\Delta_{i}\right)$, is not an orthogonal projection operator for the scalar product of $L^{2}\left(\Delta_{i}\right)$. Our first theorem establishes an $L^{p}$-error estimate for $r_{i}$.

THEOREM 2.1. Assume that $\left(\mathcal{T}_{h}\right)_{h}$ is a regular family of triangulations. For any integers $k$ and $\ell$ with $k \geq 1$ and $0 \leq \ell \leq k+1$ and any number $p$ with $1 \leq p \leq \infty$, there exists a constant $C$, independent of $h$, such that, for any macroelement $\Delta_{i}$, any triangle $T$ contained in $\Delta_{i}$, and any function $u$ in $W^{\ell, p}\left(\Delta_{i}\right)$, the following inequality holds:

$$
\left\|u-r_{i}(u)\right\|_{L^{p}(T)} \leq C h_{T}^{\ell}|u|_{W^{\ell, p}\left(\Delta_{i}\right)} .
$$

Proof. The discussion depends upon the value of $\ell$. First suppose that $\ell$ is equal to zero, i.e., that $u$ belongs to $L^{p}\left(\Delta_{i}\right)$. Let us fix a triangle $T_{0}$ in $\Delta_{i}$; we have

$$
\left\|u-r_{i}(u)\right\|_{L^{p}\left(T_{0}\right)}=\left|\operatorname{det}\left(B_{T_{0}}\right)\right|^{1 / p}\|\hat{u}-\hat{r}(\hat{u})\|_{L^{p}(\hat{T})} \leq\left|\operatorname{det}\left(B_{T_{0}}\right)\right|^{1 / p}\|\hat{u}-\hat{r}(\hat{u})\|_{L^{p}(\hat{\Delta})} .
$$

But by the definition (2.7),

$$
\|\hat{r}(\hat{u})\|_{L^{2}(\hat{\Delta})}^{2} \leq\|\hat{u}\|_{L^{p}(\hat{\Delta})}\|\hat{r}(\hat{u})\|_{L^{p^{\prime}(\hat{\Delta})}},
$$

where $p^{\prime}$ denotes the dual exponent of $p$ :

$$
\frac{1}{p}+\frac{1}{p^{\prime}}=1 .
$$

Note that all norms are equivalent on the finite element space $\Theta(\hat{\Delta})$, since it has a finite dimension, and that the equivalence constants are bounded by a fixed constant (as $\hat{\Delta}$ can only take a fixed number of configurations). Therefore, since $\hat{r}(\hat{u})$ belongs to $\Theta(\hat{\Delta})$, for each number $p$ with $1 \leq p \leq \infty$, there exist positive constants $\hat{c}_{p}$ and $\hat{C}_{p}$, which depend only on $p$ and the dimension of $\Theta(\hat{\Delta})$, such that

$$
\hat{c}_{p}\|\hat{r}(\hat{u})\|_{L^{p}(\hat{\Delta})} \leq\|\hat{r}(\hat{u})\|_{L^{2}(\hat{\Delta})} \leq \hat{C}_{p}\|\hat{r}(\hat{u})\|_{L^{p}(\hat{\Delta})} .
$$

Hence,

$$
\|\hat{r}(\hat{u})\|_{L^{p}(\hat{\Delta})} \leq \frac{1}{\hat{c}_{p^{\prime}} \hat{c}_{p}}\|\hat{u}\|_{L^{p}(\hat{\Delta})}
$$

which proves that $\hat{r}$ is stable in $L^{p}(\hat{\Delta})$ for all $p$ with $1 \leq p \leq \infty$. As a consequence,

$$
\begin{aligned}
\left\|u-r_{i}(u)\right\|_{L^{p}\left(T_{0}\right)} & \leq\left|\operatorname{det}\left(B_{T_{0}}\right)\right|^{1 / p}\left(\|\hat{u}\|_{L^{p}(\hat{T})}+\frac{1}{\hat{c}_{p^{\prime}} \hat{c}_{p}}\|\hat{u}\|_{L^{p}(\hat{\Delta})}\right) \\
& \leq\left(1+\frac{1}{\hat{c}_{p^{\prime}} \hat{c}_{p}}\right)\left|\operatorname{det}\left(B_{T_{0}}\right)\right|^{1 / p}\|\hat{u}\|_{L^{p}(\hat{\Delta})} .
\end{aligned}
$$

But

$$
\|\hat{u}\|_{L^{p}(\hat{\Delta})}=\left(\sum_{T \subset \Delta} \frac{1}{\left|\operatorname{det}\left(B_{T}\right)\right|}\|u\|_{L^{p}(T)}^{p}\right)^{1 / p} .
$$


Therefore, using (2.4) and the definition of $\sigma_{\Delta_{i}}$, we obtain

$$
\left\|u-r_{i}(u)\right\|_{L^{p}\left(T_{0}\right)} \leq \hat{C}_{1}\left(1+\frac{1}{\hat{c}_{p^{\prime}} \hat{c}_{p}}\right) \sigma_{\Delta_{i}}^{2 / p}\|u\|_{L^{p}\left(\Delta_{i}\right)} .
$$

Now, consider the case where $\ell$ is equal to one, and take $u$ in $W^{1, p}\left(\Delta_{i}\right)$. As $\hat{r}$ is a projection, observe that

$$
\forall \hat{\theta} \in \Theta(\hat{\Delta}), \hat{u}-\hat{r}(\hat{u})=\hat{u}-\hat{\theta}-\hat{r}(\hat{u}-\hat{\theta}) .
$$

Therefore, (2.11) yields, for all numbers $p, 1 \leq p \leq \infty$,

$$
\forall \hat{\theta} \in \Theta(\hat{\Delta}),\|\hat{u}-\hat{r}(\hat{u})\|_{L^{p}(\hat{\Delta})} \leq\left(1+\frac{1}{\hat{c}_{p^{\prime}} \hat{c}_{p}}\right)\|\hat{u}-\hat{\theta}\|_{L^{p}(\hat{\Delta})} .
$$

Let $\hat{\theta}$ run through the constant functions on $\hat{\Delta}$. Then Theorem 1.1 with $k=0$ and (2.13) give

$$
\|\hat{u}-\hat{r}(\hat{u})\|_{L^{p}(\hat{\Delta})} \leq \hat{C}_{2}\left(1+\frac{1}{\hat{c}_{p^{\prime}} \hat{c}_{p}}\right)|\hat{u}|_{W^{1, p}(\hat{\Delta})}
$$

where the constant $\hat{C}_{2}$ depends only on $\hat{\Delta}$. But

$$
|\hat{u}|_{W^{1, p}(\hat{\Delta})} \leq\left(\sum_{T \subset \Delta} \frac{\left\|B_{T}\right\|^{p}}{\left|\operatorname{det}\left(B_{T}\right)\right|}|u|_{W^{1, p}(T)}^{p}\right)^{1 / p} .
$$

Therefore,

$$
\left\|u-r_{i}(u)\right\|_{L^{p}\left(T_{0}\right)} \leq \hat{C}_{3} \sigma_{\Delta_{i}}^{2 / p} h_{\Delta_{i}}|u|_{W^{1, p}\left(\Delta_{i}\right)} .
$$

Finally, let $\ell$ be $\geq 2$ and take $u$ in $W^{\ell, p}\left(\Delta_{i}\right)$. Then $\hat{u}$ is continuous, and we can choose in (2.13) $\hat{\theta}$ equal to $\hat{I}(\hat{u})$, the standard interpolant of $\hat{u}$ in $\Theta(\hat{\Delta})$. Furthermore, $\hat{u}$ belongs to $W^{\ell, p}(\hat{T})$ for all $\hat{T}$ contained in $\hat{\Delta}$, and as $\ell \leq k+1$, it follows from Theorem 1.1 that

$$
\|\hat{u}-\hat{I}(\hat{u})\|_{L^{p}(\hat{\Delta})}=\left(\sum_{\hat{T} \subset \hat{\Delta}}\|\hat{u}-\hat{I}(\hat{u})\|_{L^{p}(\hat{T})}^{p}\right)^{1 / p} \leq \hat{C}_{4}\left(\sum_{\hat{T} \subset \hat{\Delta}}|\hat{u}|_{W^{\ell, p}(\hat{T})}^{p}\right)^{1 / p} .
$$

Hence, we easily derive from (2.13) that

$$
\left\|u-r_{i}(u)\right\|_{L^{p}\left(T_{0}\right)} \leq \hat{C}_{5} \sigma_{\Delta_{i}}^{2 / p} h_{\Delta_{i}}^{\ell}\left(\sum_{T \subset \Delta_{i}}|u|_{W^{\ell, p}(T)}^{p}\right)^{1 / p} \leq \hat{C}_{5} \sigma_{\Delta_{i}}^{2 / p} h_{\Delta_{i}}^{\ell}|u|_{W^{\ell, p}\left(\Delta_{i}\right)},
$$

since $u$ belongs to $W^{\ell, p}\left(\Delta_{i}\right)$.

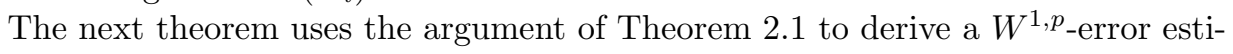
mate for $r_{i}$.

THEOREM 2.2. Assume that $\left(\mathcal{T}_{h}\right)_{h}$ is a regular family of triangulations. For any integers $k$ and $\ell$ with $k \geq 1$ and $1 \leq \ell \leq k+1$ and any number $p$ with $1 \leq p \leq \infty$, there exists a constant $C$, independent of $h$, such that, for any macroelement $\Delta_{i}$, any triangle $T$ contained in $\Delta_{i}$, and any function $u$ in $W^{\ell, p}\left(\Delta_{i}\right)$, we have

$$
\left|u-r_{i}(u)\right|_{W^{1, p}(T)} \leq C h_{T}^{\ell-1}|u|_{W^{\ell, p}\left(\Delta_{i}\right)} .
$$


Proof. Here again, the discussion depends upon the value of $\ell$. Take first $\ell$ equal to one, and $u$ in $W^{1, p}\left(\Delta_{i}\right)$. We have

$$
\left|u-r_{i}(u)\right|_{W^{1, p}\left(T_{0}\right)} \leq\left|\operatorname{det}\left(B_{T_{0}}\right)\right|^{1 / p}\left\|B_{T_{0}}^{-1}\right\||\hat{u}-\hat{r}(\hat{u})|_{W^{1, p}(\hat{\Delta})} .
$$

For any $\hat{\theta}$ in $\Theta(\hat{\Delta})$, we can write

$|\hat{u}-\hat{r}(\hat{u})|_{W^{1, p}(\hat{\Delta})} \leq|\hat{u}-\hat{\theta}|_{W^{1, p}(\hat{\Delta})}+|\hat{r}(\hat{u}-\hat{\theta})|_{W^{1, p}(\hat{\Delta})} \leq|\hat{u}-\hat{\theta}|_{W^{1, p}(\hat{\Delta})}+\hat{C}_{1}\|\hat{r}(\hat{u}-\hat{\theta})\|_{L^{p}(\hat{\Delta})}$

because all norms are equivalent on $\Theta(\hat{\Delta})$ and the equivalence constant $\hat{C}_{1}$ depends only on $\hat{\Delta}$. Then (2.11) implies that

$$
\forall \hat{\theta} \in \Theta(\hat{\Delta}),|\hat{u}-\hat{r}(\hat{u})|_{W^{1, p}(\hat{\Delta})} \leq\left(1+\frac{\hat{C}_{1}}{\hat{c}_{p^{\prime}} \hat{c}_{p}}\right)\|\hat{u}-\hat{\theta}\|_{W^{1, p}(\hat{\Delta})} .
$$

As previously, letting $\hat{\theta}$ run through the constant functions yields

$$
|\hat{u}-\hat{r}(\hat{u})|_{W^{1, p}(\hat{\Delta})} \leq \hat{C}_{2}|\hat{u}|_{W^{1, p}(\hat{\Delta})} .
$$

Therefore,

$$
\left|u-r_{i}(u)\right|_{W^{1, p}\left(T_{0}\right)} \leq \hat{C}_{2}\left|\operatorname{det}\left(B_{T_{0}}\right)\right|^{1 / p}|| B_{T_{0}}^{-1} \||\hat{u}|_{W^{1, p}(\hat{\Delta})} \leq \hat{C}_{3} \sigma_{\Delta_{i}}^{1+2 / p}|u|_{W^{1, p}\left(\Delta_{i}\right)} .
$$

When $\ell$ is $\geq 2$, we choose $\hat{\theta}=\hat{I}(\hat{u})$ in $(2.17)$. This choice gives

$$
|\hat{u}-\hat{r}(\hat{u})|_{W^{1, p}(\hat{\Delta})} \leq \hat{C}_{4}\left(\sum_{\hat{T} \subset \hat{\Delta}}|\hat{u}|_{W^{\ell, p}(\hat{T})}^{p}\right)^{1 / p} .
$$

Therefore,

$$
\left|u-r_{i}(u)\right|_{W^{1, p}\left(T_{0}\right)} \leq \hat{C}_{5} \sigma_{\Delta_{i}}^{1+2 / p} h_{\Delta_{i}}^{\ell-1}|u|_{W^{\ell, p}\left(\Delta_{i}\right)} .
$$

This proves the theorem.

Observe that when $\ell$ is $\geq 2$, the $W^{\ell, p}$-norm of $\hat{u}$ is never taken on $\hat{\Delta}$ but only separately on each $\hat{T}$. The reason for this is that, although $u$ belongs to $W^{\ell, p}\left(\Delta_{i}\right)$, $\hat{u}=u \circ F_{i}$ does not belong, in general, to $W^{\ell, p}(\hat{\Delta})$. This lack of regularity explains why $\hat{\theta}$ is chosen in the local finite element space $\Theta(\hat{\Delta})$ and not in $\mathbb{P}_{k}$. In fact, the following counterexample shows that this last choice does not yield the estimates of Theorems 2.1 and 2.2 .

$A$ counterexample. Let $h$ be any positive real number; define the two consecutive intervals $I_{1}=[0,2 h]$ and $I_{2}=[2 h, 3 h]$, and set $\Delta=I_{1} \cup I_{2}$. We associate with $\Delta$ the reference macroelement $\hat{\Delta}=\hat{I}_{1} \cup \hat{I}_{2}$, where $\hat{I}_{1}=[-1,0]$ and $\hat{I}_{2}=[0,1]$. The continuous piecewise affine mapping $F$ that maps $\hat{\Delta}$ onto $\Delta$ is

$$
F(t)= \begin{cases}2 h(1+t) & \text { on } \hat{I}_{1}, \\ 2 h+h t & \text { on } \hat{I}_{2} .\end{cases}
$$

Now, consider the function $v(x)=x$, and let $p=\hat{r}(\hat{v})$ be the projection of $\hat{v}=v \circ F$ onto $\mathbb{P}_{1}$ for the $L^{2}(\hat{\Delta})$ scalar product; i.e.,

$$
\int_{-1}^{1} p(t) d t=\int_{-1}^{1}(v \circ F)(t) d t \quad \text { and } \quad \int_{-1}^{1} t p(t) d t=\int_{-1}^{1} t(v \circ F)(t) d t .
$$


An easy calculation gives

$$
p(t)=\frac{7}{4} h+\frac{3}{2} h t
$$

and

$$
(v \circ F)(t)-p(t)= \begin{cases}h\left(\frac{1}{4}+\frac{1}{2} t\right) & \text { on } \hat{I}_{1}, \\ h\left(\frac{1}{4}-\frac{1}{2} t\right) & \text { on } \hat{I}_{2} .\end{cases}
$$

Then, on one hand,

$$
\left\|v-p \circ F^{-1}\right\|_{L^{2}(\Delta)}=\frac{1}{4} h^{3 / 2},
$$

and on the other hand,

$$
|v|_{H^{2}(\Delta)}=0, \quad|v|_{H^{1}(\Delta)}=\sqrt{3 h}, \quad\|v\|_{L^{2}(\Delta)}=3 h^{3 / 2} .
$$

As a consequence,

$$
\frac{\left\|v-p \circ F^{-1}\right\|_{L^{2}(\Delta)}}{\|v\|_{H^{2}(\Delta)}}=\frac{h}{4 \sqrt{3}\left(1+3 h^{2}\right)^{1 / 2}},
$$

which is exactly of the order of $h$ and not of the order of $h^{2}$.

Remark 2. The results of this section can readily be extended to tetrahedral triangulations of three-dimensional domains with polyhedral boundaries.

Remark 3. The statement of Theorem 2.1 (resp., Theorem 2.2) extends to the case where $u$ belongs to $W^{\ell, q}\left(\Delta_{i}\right)$ for any $q$ such that $W^{\ell, q}\left(\Delta_{i}\right)$ is continuously embedded in $L^{p}\left(\Delta_{i}\right)$ (resp., $W^{1, p}\left(\Delta_{i}\right)$ ). More precisely, under the assumptions of Theorem 2.1, if $u$ belongs to $W^{\ell, q}\left(\Delta_{i}\right)$, the following bounds hold:

$$
\begin{aligned}
& \text { if } q \geq p,\left\|u-r_{i}(u)\right\|_{L^{p}\left(\Delta_{i}\right)} \leq C h_{\Delta_{i}}^{\ell} h_{\Delta_{i}}^{2 / p-2 / q}|u|_{W^{\ell, q}\left(\Delta_{i}\right)} \\
& \text { if } q<p,\left\|u-r_{i}(u)\right\|_{L^{p}\left(\Delta_{i}\right)} \leq C h_{\Delta_{i}}^{\ell} \frac{1}{\rho_{\Delta_{i}}^{2 / q-2 / p}}|u|_{W^{\ell, q}\left(\Delta_{i}\right)} .
\end{aligned}
$$

Theorem 2.2 has a similar extension. Note that these local estimates are optimal. However, for $q \geq p$, summing up the first estimate on all macroelements and using the Hölder's inequality does not lead to a global optimal estimate, while for $q<p$ summing up the second bound leads to an optimal estimate, of order $h^{\ell+2 / p-2 / q}$, thanks to the Jensen's inequality.

Remark 4. The argument used in Theorem 2.2 for proving (2.18) can be readily extended to show that

$$
|\hat{u}-\hat{r}(\hat{u})|_{W^{s, p}(\hat{\Delta})} \leq \hat{C}_{2}^{\prime}|\hat{u}|_{W^{t, p}(\hat{\Delta})},
$$

for any real numbers $s$ and $t$ with $0 \leq s \leq 1$ and $s \leq t \leq 1$. It also holds for $1<t \leq 2$ by letting $\hat{\theta}$ run through piecewise affine functions. So, the following estimate holds for any real numbers $s$ and $t$ with $0 \leq s \leq 1$ and $s \leq t \leq k+1$ and any number $p$ with $1 \leq p \leq \infty$, provided that the function $u$ belongs to $W^{t, p}\left(\Delta_{i}\right)$ :

$$
\left|u-r_{i}(u)\right|_{W^{s, p}(T)} \leq C h_{T}^{t-s}|u|_{W^{t, p}\left(\Delta_{i}\right)} .
$$




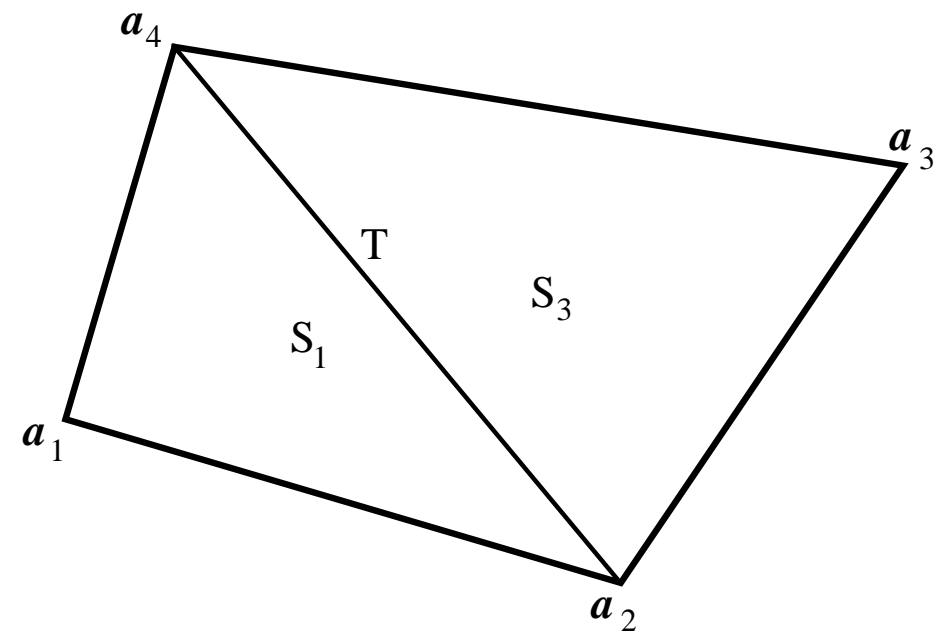

FIG. 4

This estimate can also be derived from the principal theorem of interpolation between Banach spaces (see [12, Chap. 1]) with the seminorm $|\cdot|_{W^{t, p}\left(\Delta_{i}\right)}$ replaced by the norm $\|\cdot\|_{W^{t, p}\left(\Delta_{i}\right)}$ in the right-hand side. The proof of the following result, concerning the approximation on sides $f$ of elements $T$, relies on similar arguments: for any real numbers $s, t$, and $p$ with $0 \leq s \leq 1, s+\frac{1}{p}<t \leq k+1$, and $1 \leq p<\infty$, if the function $u$ belongs to $W^{t, p}\left(\Delta_{i}\right)$, we have the estimate

$$
\left|u-r_{i}(u)\right|_{W^{s, p}(f)} \leq C h_{T}^{t-s-\frac{1}{p}}|u|_{W^{t, p}\left(\Delta_{i}\right)} .
$$

3. A projection operator on quadrilateral meshes. Let $\mathcal{T}_{h}$ be a quadrangulation of $\bar{\Omega}$ made of convex and nondegenerate quadrilaterals $T$ (i.e., not reduced to triangles) with diameter bounded by $h$. Let $T$ be one of these quadrilaterals, let $\boldsymbol{a}_{i}$ be its vertices, $1 \leq i \leq 4$, numbered counterclockwise, and let $S_{i}$ denote its subtriangle with vertices $\boldsymbol{a}_{i-1}, \boldsymbol{a}_{i}, \boldsymbol{a}_{i+1}$, the indices being numbered modulo four, as in Figure 4. Let $h_{i}$ be the diameter of $S_{i}$, and $\rho_{i}$ the diameter of its inscribed circle. We set

$$
h_{T}=\sup _{1 \leq i \leq 4} h_{i}, \quad \rho_{T}=2 \inf _{1 \leq i \leq 4} \rho_{i}, \quad \text { and } \quad \sigma_{T}=\frac{h_{T}}{\rho_{T}} .
$$

Clearly, $h_{T}$ is the diameter of $T$, and $\sigma_{T}$ is a measure of the nondegeneracy of $T$. Here also, we assume that the family of quadrangulations $\left(\mathcal{T}_{h}\right)_{h}$ is regular, i.e., there exists a constant $\sigma$, independent of $h$, such that

$$
\forall T \in \mathcal{T}_{h}, \sigma_{T} \leq \sigma .
$$

In contrast to triangular finite element spaces, in the case of quadrilaterals, the finite elements are defined first on the reference square $\hat{T}=[0,1] \times[0,1]$ and, after they are transformed into functions (generally, not polynomials), defined on the quadrilateral $T$ by a transformation from $T$ onto $\hat{T}$. More precisely (cf. [6]), as $T$ is convex and nondegenerate, there exists an invertible, bilinear mapping $\mathcal{F}_{T}$ (i.e., with components in $\left.\mathbb{Q}_{1}\right)$ that maps $\hat{T}$ onto $T$ with $\boldsymbol{a}_{i}=\mathcal{F}_{T}\left(\hat{\boldsymbol{a}}_{i}\right), 1 \leq i \leq 4$, where $\hat{\boldsymbol{a}}_{1}=(0,0), \hat{\boldsymbol{a}}_{2}=(1,0), \hat{\boldsymbol{a}}_{3}=(1,1)$, and $\hat{\boldsymbol{a}}_{4}=(0,1)$ are the vertices of $\hat{T}$. Let $D \mathcal{F}_{T}$ 
and $J_{T}$ (resp., $D \mathcal{F}_{T}^{-1}$ and $J_{T}^{-1}$ ) denote the Jacobian matrix and the Jacobian of $\mathcal{F}_{T}$ (resp., $\left.\mathcal{F}_{T}^{-1}\right)$. In the case of quadrilaterals none of these quantities are constant, but they satisfy the following bounds:

$$
\begin{gathered}
\left\|J_{T}\right\|_{L^{\infty}(\hat{T})}=2 \sup _{1 \leq i \leq 4}\left|S_{i}\right| \leq \frac{\sqrt{3}}{2} h_{T}^{2}, \quad\left\|J_{T}^{-1}\right\|_{L^{\infty}(T)}=\frac{1}{2 \inf _{1 \leq i \leq 4}\left|S_{i}\right|}<\frac{8}{\pi \rho_{T}^{2}} \\
\left\|D \mathcal{F}_{T}\right\|_{L^{\infty}(\hat{T})} \leq C_{1} h_{T}, \quad\left\|D \mathcal{F}_{T}^{-1}\right\|_{L^{\infty}(T)} \leq C_{2} \frac{\sigma_{T}}{\rho_{T}}
\end{gathered}
$$

Then we define the function space $\mathcal{Q}_{k}(T)$ by

$$
\mathcal{Q}_{k}(T)=\left\{q=\hat{q} \circ \mathcal{F}_{T}^{-1} ; \hat{q} \in \mathbb{Q}_{k}\right\} .
$$

The corresponding standard finite element space, for a positive integer $k$, is

$$
\Theta_{h}=\left\{\theta_{h} \in \mathcal{C}^{0}(\bar{\Omega}) ; \forall T \in \mathcal{T}_{h}, \theta_{h \mid T} \in \mathcal{Q}_{k}(T)\right\} .
$$

Here also, for the sake of simplicity, we assume that, in each $T$, the degrees of freedom of any function of $\Theta_{h}$ are its values at the principal lattice of order $k$. Let $N$ be the number of nodes where these degrees of freedom are defined, and let $\left\{\boldsymbol{a}_{i}, 1 \leq i \leq N\right\}$, denote this set of nodes. Here again, for any node $\boldsymbol{a}_{i}$, let the macroelement $\Delta_{i}$ be the union of the quadrilaterals of $\mathcal{T}_{h}$ that share this node $\boldsymbol{a}_{i}$, and define

$$
h_{\Delta_{i}}=\sup _{T \subset \Delta_{i}} h_{T}, \quad \rho_{\Delta_{i}}=\inf _{T \subset \Delta_{i}} \rho_{T}, \quad \sigma_{\Delta_{i}}=\frac{h_{\Delta_{i}}}{\rho_{\Delta_{i}}} .
$$

If the mesh is Cartesian, the situation is simpler than that of the previous section, because for all nodes $\boldsymbol{a}_{i}, \Delta_{i}$ consists of one, two, or four quadrilaterals (or possibly three if $\boldsymbol{a}_{i}$ is a boundary node) and the reference macroelement associated with any $\Delta_{i}$ is made of at most four unit squares. But we do not necessarily choose a Cartesian mesh, and at a node where the mesh is not Cartesian, the reference macroelement cannot consist of unit squares. Indeed, let $\boldsymbol{a}_{i}$ denote a node where the mesh is not Cartesian, and suppose that the corresponding macroelement $\Delta_{i}$ has $J$ elements. Consider one element $T$ in $\Delta_{i}$, and to simplify the discussion, let $\boldsymbol{a}_{i}=\boldsymbol{a}_{1}$, as in the example of Figure 5 , and let $S=S_{1}$ and $S^{\prime}=S_{3}$ be the two corresponding subtriangles of $T$. Let $D_{i}$ be the auxiliary macroelement consisting of all these subtriangles $S$ with common vertex $\boldsymbol{a}_{i}$, and let $\tilde{D}_{i}$ be the corresponding auxiliary reference macroelement consisting of $J$ equal isosceles unit triangles as in Figure 3.

Let $\tilde{S}$ be one of these triangles with vertices denoted by $\tilde{\boldsymbol{a}}_{1}=(0,0), \tilde{\boldsymbol{a}}_{2}$, and $\tilde{\boldsymbol{a}}_{4}$, as in Figure 5. Since $T$ is convex and not reduced to a triangle, there exists a unique affine invertible mapping $F_{S}$ such that $S=F_{S}(\tilde{S})$ and $\boldsymbol{a}_{i}=F_{S}\left(\tilde{\boldsymbol{a}}_{i}\right), i=1,2,4$ :

$$
\boldsymbol{x}=F_{S}(\tilde{\boldsymbol{x}})=B_{S} \tilde{\boldsymbol{x}}+\boldsymbol{a}_{1} .
$$

We construct an auxiliary reference element $\tilde{T}$ by means of the mapping $F_{S}$ in the following way. Let $\tilde{\boldsymbol{a}}_{3}=F_{S}^{-1}\left(\boldsymbol{a}_{3}\right)$, and let $\tilde{S}^{\prime}$ denote the triangle with vertices $\tilde{\boldsymbol{a}}_{2}, \tilde{\boldsymbol{a}}_{3}$, and $\tilde{\boldsymbol{a}}_{4}$. We associate with $T$ the auxiliary reference element $\tilde{T}=\tilde{S} \cup \tilde{S}^{\prime}$. Clearly, $\tilde{T}$ is also convex and not reduced to a triangle, and therefore, there exists a unique bilinear mapping $\mathcal{F}_{\tilde{T}}$ such that $\tilde{T}=\mathcal{F}_{\tilde{T}}(\hat{T})$ and $\tilde{\boldsymbol{a}}_{i}=\mathcal{F}_{\tilde{T}}\left(\hat{\boldsymbol{a}}_{i}\right), 1 \leq i \leq 4$. In fact,

$$
\mathcal{F}_{T}=F_{S} \circ \mathcal{F}_{\tilde{T}}
$$




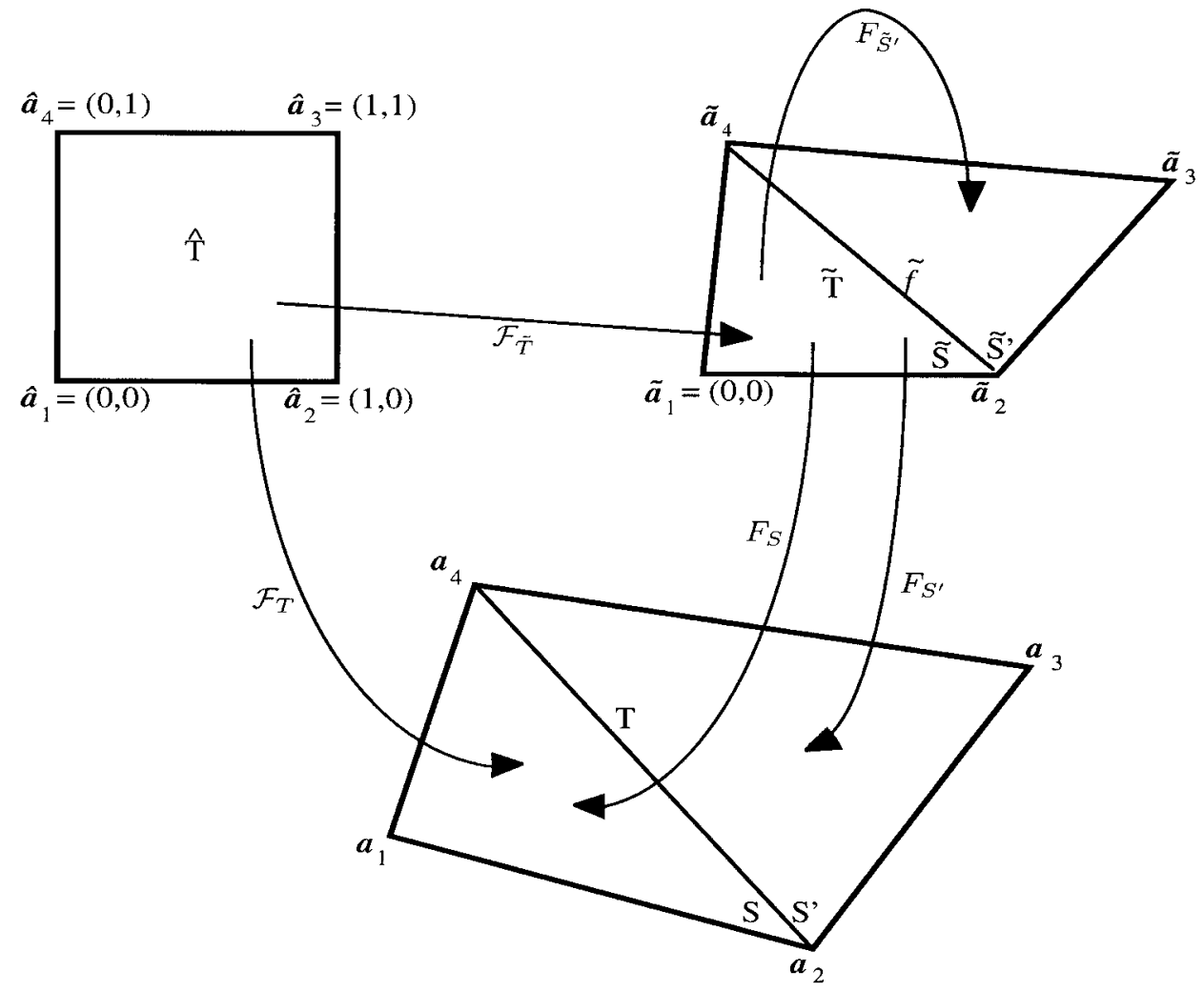

FIG. 5

Let $\tilde{D}_{i}^{\prime}$ be the union of the triangles $\tilde{S}^{\prime}$ associated with all the triangles $\tilde{S}$ in $\tilde{D}_{i}$; we take for reference macroelement

$$
\tilde{\Delta}_{i}=\tilde{D}_{i} \cup \tilde{D}_{i}^{\prime}
$$

Observe that $\tilde{\Delta}_{i}$ is a variable macroelement because the triangles $\tilde{S}^{\prime}$ constituting $\tilde{D}_{i}^{\prime}$ do not have a regular shape; as a consequence, we cannot apply directly on $\tilde{\Delta}_{i}$ any result that depends upon the shape of the domain. In order to take into account the geometry of $\tilde{D}_{i}^{\prime}$, we introduce first the affine invertible mapping $F_{\tilde{S}^{\prime}}$ that maps $\tilde{S}$ onto $\tilde{S}^{\prime}$ and leaves invariant $\tilde{f}$, the diagonal separating $\tilde{S}$ and $\tilde{S}^{\prime}$; i.e., $\tilde{S}^{\prime}=F_{\tilde{S}^{\prime}}(\tilde{S})$, $\tilde{f}=F_{\tilde{S}^{\prime}}(\tilde{f})$, and $\tilde{\boldsymbol{a}}_{3}=F_{\tilde{S}^{\prime}}\left(\tilde{\boldsymbol{a}}_{1}\right)$ :

$$
\tilde{\boldsymbol{y}}=F_{\tilde{S}^{\prime}}(\tilde{\boldsymbol{x}})=B_{\tilde{S}^{\prime}} \tilde{\boldsymbol{x}}+\tilde{\boldsymbol{a}}_{3} .
$$

And finally, let $F_{S^{\prime}}$ denote the affine invertible mapping such that $S^{\prime}=F_{S^{\prime}}(\tilde{S})$ and $\boldsymbol{a}_{3}=F_{S^{\prime}}\left(\tilde{\boldsymbol{a}}_{1}\right)$ :

$$
\boldsymbol{x}=F_{S^{\prime}}(\tilde{\boldsymbol{x}})=B_{S^{\prime}} \tilde{\boldsymbol{x}}+\boldsymbol{a}_{3} .
$$

Note that $S^{\prime}=F_{S}\left(\tilde{S}^{\prime}\right)=F_{S} \circ F_{\tilde{S}^{\prime}}(\tilde{S})$ :

$$
\boldsymbol{x}=B_{S} B_{\tilde{S}^{\prime}} \tilde{\boldsymbol{x}}+B_{S} \tilde{\boldsymbol{a}}_{3}+\boldsymbol{a}_{1}=B_{S} B_{\tilde{S}^{\prime}} \tilde{\boldsymbol{x}}+\boldsymbol{a}_{3}
$$


therefore,

$$
B_{S^{\prime}}=B_{S} B_{\tilde{S}^{\prime}}
$$

and this equality allows one to estimate the geometrical parameters related to $B_{\tilde{S}^{\prime}}$. Indeed, denoting by $h_{U}$ the diameter of any triangle $U$, by $\rho_{U}$ the diameter of the circle inscribed in $U$, and setting naturally $\sigma_{U}=h_{U} / \rho_{U}$, we have

$$
\begin{array}{ccc}
\left\|B_{S}\right\| \leq \frac{h_{S}}{\rho_{\tilde{S}}}, & \left\|B_{S}^{-1}\right\| \leq \frac{h_{\tilde{S}}}{\rho_{S}}, & \left|\operatorname{det}\left(B_{S}\right)\right|=\frac{|S|}{|\tilde{S}|}, \\
\left\|B_{S^{\prime}}\right\| \leq \frac{h_{S^{\prime}}}{\rho_{\tilde{S}}}, & \left\|B_{S^{\prime}}^{-1}\right\| \leq \frac{h_{\tilde{S}}}{\rho_{S^{\prime}}}, & \left|\operatorname{det}\left(B_{S^{\prime}}\right)\right|=\frac{\left|S^{\prime}\right|}{|\tilde{S}|} .
\end{array}
$$

Thus, as $B_{\tilde{S}^{\prime}}=B_{S}^{-1} B_{S^{\prime}}$, we obtain

$$
\left\|B_{\tilde{S}^{\prime}}\right\| \leq \sigma_{\tilde{S}} \frac{h_{S^{\prime}}}{\rho_{S}} \leq 2 \sigma_{\tilde{S}} \sigma_{T},\left\|B_{\tilde{S}^{\prime}}^{-1}\right\| \leq 2 \sigma_{\tilde{S}} \sigma_{T},\left|\operatorname{det}\left(B_{\tilde{S}^{\prime}}\right)\right|=\frac{\left|S^{\prime}\right|}{|S|} \leq 4 \frac{\sqrt{3}}{\pi} \sigma_{T}^{2},
$$

and since the family of quadrangulations is regular, these three quantities can be bounded independently of $h$.

Similarly, the fact that $\mathcal{F}_{\tilde{T}}=F_{S}^{-1} \circ \mathcal{F}_{T}$, and hence $D \mathcal{F}_{\tilde{T}}=B_{S}^{-1} \cdot D \mathcal{F}_{T}$, allows one to estimate the geometrical parameters related to $\mathcal{F}_{\tilde{T}}$ :

$$
\begin{aligned}
& \left\|D \mathcal{F}_{\tilde{T}}\right\|_{L^{\infty}(\hat{T})} \leq C_{3} \sigma_{T}, \quad\left\|D \mathcal{F}_{\tilde{T}}^{-1}\right\|_{L^{\infty}(\tilde{T})} \leq C_{4} \sigma_{T}^{2}, \\
& \left\|J_{\tilde{T}}\right\|_{L^{\infty}(\hat{T})} \leq C_{5} \sigma_{T}^{2}, \quad\left\|J_{\tilde{T}}^{-1}\right\|_{L^{\infty}(\tilde{T})} \leq C_{6} \sigma_{T}^{2} .
\end{aligned}
$$

Owing to the above construction, there exists a continuous and invertible mapping $F_{i}$, that is affine on each "reference" quadrilateral $\tilde{T}$ of $\tilde{\Delta}_{i}$ and coincides with $F_{S}$ on $\tilde{T}$ :

$$
\forall \tilde{\boldsymbol{x}} \in \tilde{T}, F_{i}(\tilde{\boldsymbol{x}})=F_{S}(\tilde{\boldsymbol{x}}) .
$$

Moreover, $F_{i}$ is such that

$$
\Delta_{i}=F_{i}\left(\tilde{\Delta}_{i}\right)
$$

Since the family of quadrangulations is regular, properties (i)-(iii) of section 2 obviously hold here and, as in the preceding section, all geometric constants of $\tilde{\Delta}_{i}$ can be bounded by constants independent of $i$; therefore, we drop the index $i$. Similarly, property (iv) holds with $K=(k+1)^{2}$.

Then we associate with $\Theta_{h}$ the local finite element spaces

$$
\begin{gathered}
\Theta(\tilde{\Delta})=\left\{\tilde{\theta} \in \mathcal{C}^{0}(\tilde{\Delta}) ; \forall \tilde{T} \subset \tilde{\Delta}, \tilde{\theta}_{\mid \tilde{T}} \in \mathcal{Q}_{k}(\tilde{T})\right\}, \\
\Theta\left(\Delta_{i}\right)=\left\{\theta \in \mathcal{C}^{0}\left(\Delta_{i}\right) ; \forall T \subset \Delta_{i}, \theta_{\mid T} \in \mathcal{Q}_{k}(T)\right\},
\end{gathered}
$$

and we define the projection operator $\tilde{r}$ in analogy to the preceding section. More precisely, for any function $\tilde{u}$ in $L^{1}(\tilde{\Delta})$, we define $\tilde{r}(\tilde{u})$ in $\Theta(\tilde{\Delta})$ by

$$
\forall \tilde{\theta} \in \Theta(\tilde{\Delta}), \int_{\tilde{\Delta}}(\tilde{r}(\tilde{u})-\tilde{u}) \tilde{\theta} d \tilde{\boldsymbol{x}}=0
$$


and for any function $u$ in $L^{1}\left(\Delta_{i}\right)$, we define $r_{i}(u)$ in $\Theta\left(\Delta_{i}\right)$ by

$$
r_{i}(u) \circ F_{i}=\tilde{r}\left(u \circ F_{i}\right),
$$

which we denote symbolically by $\widetilde{r_{i}(u)}=\tilde{r}(\tilde{u})$.

Looking back at the proofs of the previous section, we see that we need two equivalences of norms satisfied by functions of $\Theta(\tilde{\Delta})$, and we think of applying Theorem 1.1 on $\tilde{\Delta}$ (observe that Theorem 1.1 is relevant here because the mapping $F_{i}$ is piecewise affine). But since $\tilde{\Delta}$ is composed of variable quadrilaterals, these equivalences are no longer simple consequences of the finite dimension of $\Theta(\tilde{\Delta})$, and neither does Theorem 1.1 apply directly on $\tilde{\Delta}$. These results are established in the next three lemmas.

Lemma 3.1. Assume that $\left(\mathcal{T}_{h}\right)_{h}$ is a regular family of quadrangulations. For each number $p$ with $1 \leq p \leq \infty$, there exist positive constants $\hat{c}_{p}$ and $\hat{C}_{p}$, independent of $h$, such that, for all $\tilde{\Delta}$, we have the following equivalence:

$$
\forall \tilde{\theta} \in \Theta(\tilde{\Delta}), \hat{c}_{p}\|\tilde{\theta}\|_{L^{p}(\tilde{\Delta})} \leq\|\tilde{\theta}\|_{L^{2}(\tilde{\Delta})} \leq \hat{C}_{p}\|\tilde{\theta}\|_{L^{p}(\tilde{\Delta})} .
$$

Proof. We have

$$
\begin{aligned}
\|\tilde{\theta}\|_{L^{p}(\tilde{\Delta})} & =\left(\sum_{\tilde{T} \subset \tilde{\Delta}}\|\tilde{\theta}\|_{L^{p}(\tilde{T})}^{p}\right)^{1 / p} \leq\left(\sum_{\tilde{T} \subset \tilde{\Delta}}\left\|J_{\tilde{T}}\right\|_{L^{\infty}(\hat{T})}\left\|\tilde{\theta} \circ \mathcal{F}_{\tilde{T}}\right\|_{L^{p}(\hat{T})}^{p}\right)^{1 / p} \\
& \leq \hat{C}_{1} \sigma_{\Delta_{i}}^{2 / p}\left(\sum_{\tilde{T} \subset \tilde{\Delta}}\left\|\tilde{\theta} \circ \mathcal{F}_{\tilde{T}}\right\|_{L^{p}(\hat{T})}^{p}\right)^{1 / p}
\end{aligned}
$$

by applying (3.6). But since $\tilde{\theta} \circ \mathcal{F}_{\tilde{T}}$ belongs to the finite-dimensional space $\mathbb{Q}_{k}$ on the reference square $\hat{T}$, there exists a constant $\hat{C}_{2}$ such that

$$
\left\|\tilde{\theta} \circ \mathcal{F}_{\tilde{T}}\right\|_{L^{p}(\hat{T})} \leq \hat{C}_{2}\left\|\tilde{\theta} \circ \mathcal{F}_{\tilde{T}}\right\|_{L^{2}(\hat{T})} .
$$

Thus, reverting to each $\tilde{T}$ and applying again (3.6), we obtain

$$
\|\tilde{\theta}\|_{L^{p}(\tilde{\Delta})} \leq \hat{C}_{3} \sigma_{\Delta_{i}}^{2 / p+1}\|\tilde{\theta}\|_{L^{2}(\tilde{\Delta})} .
$$

This establishes the first part of (3.11). The proof of the second part is similar.

Lemma 3.2. Assume that $\left(\mathcal{T}_{h}\right)_{h}$ is a regular family of quadrangulations. For each number $p$ with $1 \leq p \leq \infty$, there exists a positive constant $\hat{C}_{p}$, independent of $h$, such that, for all $\tilde{\Delta}$, we have the following inverse inequality:

$$
\forall \tilde{\theta} \in \Theta(\tilde{\Delta}),|\tilde{\theta}|_{W^{1, p}(\tilde{\Delta})} \leq \hat{C}_{p}\|\tilde{\theta}\|_{L^{p}(\tilde{\Delta})} .
$$

Proof. We have

$$
|\tilde{\theta}|_{W^{1, p}(\tilde{\Delta})} \leq\left(\sum_{\tilde{T} \subset \tilde{\Delta}}\left\|J_{\tilde{T}}\right\|_{L^{\infty}(\hat{T})}\left\|D \mathcal{F}_{\tilde{T}}\right\|_{L^{\infty}(\hat{T})}^{p}\left|\tilde{\theta} \circ \mathcal{F}_{\tilde{T}}\right|_{W^{1, p}(\hat{T})}^{p}\right)^{1 / p} .
$$


Again, since $\tilde{\theta} \circ \mathcal{F}_{\tilde{T}}$ belongs to the finite-dimensional space $\mathbb{Q}_{k}$ on the reference square $\hat{T}$, there exists a constant $\hat{C}_{1}$ such that

$$
\left|\tilde{\theta} \circ \mathcal{F}_{\tilde{T}}\right|_{W^{1, p}(\hat{T})} \leq \hat{C}_{1}\left\|\tilde{\theta} \circ \mathcal{F}_{\tilde{T}}\right\|_{L^{p}(\hat{T})} .
$$

Therefore, in view of (3.6), we obtain

$$
|\tilde{\theta}|_{W^{1, p}(\tilde{\Delta})} \leq \hat{C}_{2} \sigma_{\Delta_{i}}^{4 / p+1}\|\tilde{\theta}\|_{L^{p}(\tilde{\Delta})},
$$

thus proving (3.12).

The next result is a special case of some inequalities of [9] and [10]; we give the proof for the sake of completeness.

Lemma 3.3. Assume that $\left(\mathcal{T}_{h}\right)_{h}$ is a regular family of quadrangulations. For any function $\tilde{\theta}$ in $L^{1}(\tilde{\Delta})$, define the average

$$
c(\tilde{\theta})=\frac{1}{|\tilde{D}|} \int_{\tilde{D}} \tilde{\theta} d \tilde{\boldsymbol{x}} .
$$

For each number $p$ with $1 \leq p \leq \infty$, there exists a positive constant $\hat{C}_{p}$, independent of $h$, such that for all $\tilde{\Delta}$, we have

$$
\forall \tilde{\theta} \in W^{1, p}(\tilde{\Delta}),\|\tilde{\theta}-c(\tilde{\theta})\|_{L^{p}(\tilde{\Delta})} \leq \hat{C}_{p}|\tilde{\theta}|_{W^{1, p}(\tilde{\Delta})} .
$$

Proof. We write

$$
\|\tilde{\theta}-c(\tilde{\theta})\|_{L^{p}(\tilde{\Delta})} \leq\left(\|\tilde{\theta}-c(\tilde{\theta})\|_{L^{p}(\tilde{D})}^{p}+\|\tilde{\theta}-c(\tilde{\theta})\|_{L^{p}\left(\tilde{D}^{\prime}\right)}^{p}\right)^{1 / p} .
$$

Since $\tilde{D}$ has a regular shape that can assume only a fixed number of configurations, and $c(\tilde{\theta})=\tilde{\theta}$ when $\tilde{\theta}$ is a constant function, we can apply Theorem 1.1 with $k=0$ on $\tilde{D}$ : there exists a constant $\hat{C}_{1}$, independent of $\tilde{D}$, such that

$$
\|\tilde{\theta}-c(\tilde{\theta})\|_{L^{p}(\tilde{D})} \leq \hat{C}_{1}|\tilde{\theta}|_{W^{1, p}(\tilde{D})} .
$$

It remains to estimate the second term of (3.14). Consider a triangle $\tilde{S}^{\prime}$ in $\tilde{D}^{\prime}$, and let us switch to $\tilde{S}$ :

$$
\|\tilde{\theta}-c(\tilde{\theta})\|_{L^{p}\left(\tilde{S}^{\prime}\right)}=\left|\operatorname{det}\left(B_{\tilde{S}^{\prime}}\right)\right|^{1 / p}\left\|\tilde{\theta} \circ F_{\tilde{S}^{\prime}}-c(\tilde{\theta})\right\|_{L^{p}(\tilde{S})} .
$$

But since $\tilde{S}$ has a regular shape, there exists a constant $\hat{C}_{2}$ such that

$$
\forall v \in W^{1, p}(\tilde{S}),\|v\|_{W^{1, p}(\tilde{S})} \leq \hat{C}_{2}\left(|v|_{W^{1, p}(\tilde{S})}^{p}+\|v\|_{L^{p}(\tilde{f})}^{p}\right)^{1 / p} .
$$

Therefore,

$$
\left\|\tilde{\theta} \circ F_{\tilde{S}^{\prime}}-c(\tilde{\theta})\right\|_{L^{p}(\tilde{S})} \leq \hat{C}_{2}\left(\left|\tilde{\theta} \circ F_{\tilde{S}^{\prime}}^{\prime}\right|_{W^{1, p}(\tilde{S})}^{p}+\left\|\tilde{\theta} \circ F_{\tilde{S}^{\prime}}-c(\tilde{\theta})\right\|_{L^{p}(\tilde{f})}^{p}\right)^{1 / p} .
$$

On one hand, for any function $v, v \circ F_{\tilde{S}^{\prime}}$ coincides with $v$ on $\tilde{f}$ because $F_{\tilde{S}^{\prime}}$ reduces to the identity mapping on $\tilde{f}$, and hence,

$$
\left\|\tilde{\theta} \circ F_{\tilde{S}^{\prime}}-c(\tilde{\theta})\right\|_{L^{p}(\tilde{f})}=\|\tilde{\theta}-c(\tilde{\theta})\|_{L^{p}(\tilde{f})} \leq \hat{C}_{3}\|\tilde{\theta}-c(\tilde{\theta})\|_{W^{1, p}(\tilde{S})}
$$


by applying a trace theorem on $\tilde{S}$. On the other hand, reverting to $\tilde{S}^{\prime}$,

$$
\left|\tilde{\theta} \circ F_{\tilde{S}^{\prime}}\right|_{W^{1, p}(\tilde{S})} \leq\left|\operatorname{det}\left(B_{\tilde{S}^{\prime}}\right)\right|^{-1 / p} \| B_{\tilde{S}^{\prime}}|||\tilde{\theta}|_{W^{1, p}\left(\tilde{S}^{\prime}\right)} \leq 2 \sigma_{\tilde{S}^{\prime}} \sigma_{T}\left|\operatorname{det}\left(B_{\tilde{S}^{\prime}}\right)\right|^{-1 / p}|\tilde{\theta}|_{W^{1, p}\left(\tilde{S}^{\prime}\right)} \text {. }
$$

Hence,

$$
\|\tilde{\theta}-c(\tilde{\theta})\|_{L^{p}\left(\tilde{S}^{\prime}\right)} \leq \hat{C}_{4}\left(\sigma_{T}^{p}|\tilde{\theta}|_{W^{1, p}\left(\tilde{S}^{\prime}\right)}^{p}+\left|\operatorname{det}\left(B_{\tilde{S}^{\prime}}\right)\right|\|\tilde{\theta}-c(\tilde{\theta})\|_{W^{1, p}(\tilde{S})}^{p}\right)^{1 / p},
$$

and summing over all triangles of $\tilde{D}^{\prime}$,

$$
\|\tilde{\theta}-c(\tilde{\theta})\|_{L^{p}\left(\tilde{D}^{\prime}\right)} \leq \hat{C}_{5}\left(\sigma_{\Delta_{i}}^{p}|\tilde{\theta}|_{W^{1, p}\left(\tilde{D}^{\prime}\right)}^{p}+\sigma_{\Delta_{i}}^{2}\|\tilde{\theta}-c(\tilde{\theta})\|_{W^{1, p}(\tilde{D})}^{p}\right)^{1 / p} .
$$

Then (3.15) gives

$$
\|\tilde{\theta}-c(\tilde{\theta})\|_{L^{p}\left(\tilde{D}^{\prime}\right)} \leq \hat{C}_{6} \sigma_{\Delta_{i}}^{\max (2 / p, 1)}|\tilde{\theta}|_{W^{1, p}(\tilde{\Delta})},
$$

thus proving (3.13).

The following theorems are analogues of Theorems 2.1 and 2.2. We skip their proofs because, owing to Lemmas 3.1, 3.2, and 3.3, they are very similar to those of Theorems 2.1 and 2.2 .

THEOREM 3.4. Assume that $\left(\mathcal{T}_{h}\right)_{h}$ is a regular family of quadrangulations. For any integers $k$ and $\ell$ with $k \geq 1$ and $0 \leq \ell \leq k+1$ and any number $p$ with $1 \leq p \leq \infty$, there exists a constant $C$, independent of $h$, such that, for any macroelement $\Delta_{i}$, any quadrilateral $T$ contained in $\Delta_{i}$, and any function $u$ in $W^{\ell, p}\left(\Delta_{i}\right)$, the following inequality holds:

$$
\left\|u-r_{i}(u)\right\|_{L^{p}(T)} \leq C h_{T}^{\ell}|u|_{W^{\ell, p}\left(\Delta_{i}\right)} .
$$

TheOREM 3.5. Assume that $\left(\mathcal{T}_{h}\right)_{h}$ is a regular family of quadrangulations. For any integers $k$ and $\ell$ with $k \geq 1$ and $1 \leq \ell \leq k+1$ and any number $p$ with $1 \leq p \leq \infty$, there exists a constant $C$, independent of $h$, such that, for any macroelement $\Delta_{i}$, any quadrilateral $T$ contained in $\Delta_{i}$, and any function $u$ in $W^{\ell, p}\left(\Delta_{i}\right)$, we have

$$
\left|u-r_{i}(u)\right|_{W^{1, p}(T)} \leq C h_{T}^{\ell-1}|u|_{W^{\ell, p}\left(\Delta_{i}\right)} .
$$

Remark 5. Similar arguments to those of section 2 yield that, under the same assumptions, estimates (2.19) to (2.21) still hold for quadrilateral meshes.

4. A regularizing operator. We shall first study the regularization of functions with no imposed value on the boundary. Let $\mathcal{T}_{h}$ be a triangulation or quadrangulation of $\bar{\Omega}$ as defined in sections 2 or 3 , let $\Theta_{h}$ be the finite element space defined by (2.1) or (3.3) for some positive integer $k$, and let $\left\{\boldsymbol{a}_{i}, 1 \leq i \leq N\right\}$ denote the set of nodes of $\mathcal{T}_{h}$ where the degrees of freedom of the functions of $\Theta_{h}$ are defined. For $1 \leq i \leq N$, let $\varphi_{i}$ denote the basis function of $\Theta_{h}$ that takes the value one at the node $\boldsymbol{a}_{i}$ and zero at all other nodes.

For any number $p$ with $1 \leq p \leq \infty$ and any nonnegative integer $\ell$, let $u$ be a given function in $W^{\ell, p}(\Omega)$, and for any integer $i$ with $1 \leq i \leq N$, let $r_{i}(u)$ be defined by (2.8) or (3.10). Then we define the regularizing operator $R_{h}$ from $W^{\ell, p}(\Omega)$ into $\Theta_{h}$ by

$$
\forall u \in W^{\ell, p}(\Omega), R_{h}(u)(\boldsymbol{x})=\sum_{i=1}^{N}\left[r_{i}(u)\right]\left(\boldsymbol{a}_{i}\right) \varphi_{i}(\boldsymbol{x}) .
$$


Clearly $R_{h}$ is continuous from $W^{\ell, p}(\Omega)$ into $\Theta_{h}$. The next two theorems establish error estimates satisfied by $R_{h}$.

THEOREM 4.1. Assume that $\left(\mathcal{T}_{h}\right)_{h}$ is a regular family of triangulations or quadrangulations of $\bar{\Omega}$. For any integers $k$ and $\ell$ with $k \geq 1$ and $0 \leq \ell \leq k+1$ and any number $p$ with $1 \leq p \leq \infty$, there exists a constant $C$, independent of $h$, such that

$$
\forall u \in W^{\ell, p}(\Omega), \forall T \in \mathcal{T}_{h},\left\|u-R_{h}(u)\right\|_{L^{p}(T)} \leq C h_{T}^{\ell}|u|_{W^{\ell, p}\left(\Delta_{T}\right)},
$$

where $\Delta_{T}$ denotes the union of all elements in $\mathcal{T}_{h}$ which share at least a corner with $T$.

Proof. Suppose that $\Delta_{T}$ contains $n$ macroelements $\Delta_{i}$, which, for the sake of simplicity, we number from 1 to $n$. Then, owing to the support of the basis functions $\varphi_{i}$, we have

$$
\left.R_{h}(u)\right|_{T}=\left.\sum_{i=1}^{n}\left[r_{i}(u)\right]\left(\boldsymbol{a}_{i}\right) \varphi_{i}\right|_{T}
$$

Therefore, we can write

$$
\left.\left[u-R_{h}(u)\right]\right|_{T}=\left.u\right|_{T}-\left.\sum_{i=1}^{n}\left[r_{1}(u)\right]\left(\boldsymbol{a}_{i}\right) \varphi_{i}\right|_{T}-\left.\sum_{i=2}^{n}\left[r_{i}(u)-r_{1}(u)\right]\left(\boldsymbol{a}_{i}\right) \varphi_{i}\right|_{T} .
$$

But

$$
\left.\sum_{i=1}^{n}\left[r_{1}(u)\right]\left(\boldsymbol{a}_{i}\right) \varphi_{i}\right|_{T}=\left.r_{1}(u)\right|_{T}
$$

hence,

$$
\left.\left[u-R_{h}(u)\right]\right|_{T}=\left.\left[u-r_{1}(u)\right]\right|_{T}-\left.\sum_{i=2}^{n}\left[r_{i}(u)-r_{1}(u)\right]\left(\boldsymbol{a}_{i}\right) \varphi_{i}\right|_{T}
$$

Therefore,

$$
\left\|u-R_{h}(u)\right\|_{L^{p}(T)} \leq\left\|u-r_{1}(u)\right\|_{L^{p}(T)}+\sum_{i=2}^{n}\left|\left[r_{i}(u)-r_{1}(u)\right]\left(\boldsymbol{a}_{i}\right)\right|\left\|\varphi_{i}\right\|_{L^{p}(T)} .
$$

The first term on the right-hand side is estimated by Theorems 2.1 or 3.4 , and it remains to evaluate the sum. On the one hand, if $T$ is a triangle,

$$
\left\|\varphi_{i}\right\|_{L^{p}(T)}=\left|\operatorname{det}\left(B_{T}\right)\right|^{1 / p}\left\|\hat{\varphi}_{i}\right\|_{L^{p}(\hat{T})}
$$

or if $T$ is a quadrilateral,

$$
\left\|\varphi_{i}\right\|_{L^{p}(T)} \leq\left\|J_{T}\right\|_{L^{\infty}(\hat{T})}^{1 / p}\left\|\hat{\varphi}_{i}\right\|_{L^{p}(\hat{T})} .
$$

In both cases, $\left\|\hat{\varphi}_{i}\right\|_{L^{p}(\hat{T})}$ is a constant independent of $T$ and $h$. Thus we have

$$
\left\|\varphi_{i}\right\|_{L^{p}(T)} \leq \hat{C}_{1}\left|\operatorname{det}\left(B_{T}\right)\right|^{1 / p} \text { or }\left\|\varphi_{i}\right\|_{L^{p}(T)} \leq \hat{C}_{2}\left\|J_{T}\right\|_{L^{\infty}(\hat{T})}^{1 / p},
$$

according to whether $T$ is a triangle or a quadrilateral. On the other hand,

$$
\begin{aligned}
\left|\left[r_{i}(u)-r_{1}(u)\right]\left(\boldsymbol{a}_{i}\right)\right| & \leq\left\|r_{i}(u)-r_{1}(u)\right\|_{L^{\infty}(T)}=\left\|\widehat{r_{i}(u)}-\widehat{r_{1}(u)}\right\|_{L^{\infty}(\hat{T})} \\
& \leq \hat{C}_{3}\left\|\widehat{r_{i}(u)}-\widehat{r_{1}(u)}\right\|_{L^{p}(\hat{T})}
\end{aligned}
$$


since $\widehat{r_{i}(u)}-\widehat{r_{1}(u)}$ belongs to a space of finite and fixed dimension on the reference set $\hat{T}$. Therefore,

$$
\left|\left[r_{i}(u)-r_{1}(u)\right]\left(\boldsymbol{a}_{i}\right)\right| \leq \hat{C}_{3}\left(\left\|\widehat{r_{i}(u)}-\hat{u}\right\|_{L^{p}(\hat{T})}+\left\|\widehat{r_{1}(u)}-\hat{u}\right\|_{L^{p}(\hat{T})}\right) .
$$

Then, if $T$ is a triangle, by virtue of Theorem 2.1 we have

$$
\begin{aligned}
\left\|\widehat{r_{i}(u)}-\hat{u}\right\|_{L^{p}(\hat{T})} & =\left|\operatorname{det}\left(B_{T}\right)\right|^{-1 / p}\left\|r_{i}(u)-u\right\|_{L^{p}(T)} \\
& \leq \hat{C}_{4} h_{T}^{\ell}\left|\operatorname{det}\left(B_{T}\right)\right|^{-1 / p}|u|_{W^{\ell, p}\left(\Delta_{i}\right)}
\end{aligned}
$$

and if $T$ is a quadrilateral, by virtue of Theorem 3.4 we have

$$
\begin{aligned}
\left\|\widehat{r_{i}(u)}-\hat{u}\right\|_{L^{p}(\hat{T})} & \leq\left\|J_{T}^{-1}\right\|_{L^{\infty}(T)}^{1 / p}\left\|r_{i}(u)-u\right\|_{L^{p}(T)} \\
& \leq \hat{C}_{5} h_{T}^{\ell}\left\|J_{T}^{-1}\right\|_{L^{\infty}(T)}^{1 / p}|u|_{W^{\ell, p}\left(\Delta_{i}\right)} .
\end{aligned}
$$

Therefore, combining (4.5) or (4.6) with (4.4), we obtain, if $T$ is a triangle,

$$
\left|\left[r_{i}(u)-r_{1}(u)\right]\left(\boldsymbol{a}_{i}\right)\right||| \varphi_{i} \|_{L^{p}(T)} \leq \hat{C}_{6} h_{T}^{\ell}\left(|u|_{W^{\ell, p}\left(\Delta_{i}\right)}+|u|_{W^{\ell, p}\left(\Delta_{1}\right)}\right),
$$

or, if $T$ is a quadrilateral,

$$
\begin{aligned}
\left|\left[r_{i}(u)-r_{1}(u)\right]\left(\boldsymbol{a}_{i}\right)\right|\left\|\varphi_{i}\right\|_{L^{p}(T)} & \leq \hat{C}_{7} h_{T}^{\ell}\left\|J_{T}\right\|_{L^{\infty}(\hat{T})}^{1 / p}\left\|J_{T}^{-1}\right\|_{L^{\infty}(T)}^{1 / p}\left(|u|_{W^{\ell, p}\left(\Delta_{i}\right)}+|u|_{W^{\ell, p}\left(\Delta_{1}\right)}\right) \\
& \leq \hat{C}_{8} \sigma_{T}^{2 / p} h_{T}^{\ell}\left(|u|_{W^{\ell, p}\left(\Delta_{i}\right)}+|u|_{W^{\ell, p}\left(\Delta_{1}\right)}\right) .
\end{aligned}
$$

This proves the theorem.

In view of Theorems 2.2 and 3.5, the argument of Theorem 4.1 can easily be extended to prove the following estimate.

THEOREM 4.2. Assume that $\left(\mathcal{T}_{h}\right)_{h}$ is a regular family of triangulations or quadrangulations of $\bar{\Omega}$. For any integers $k$ and $\ell$ with $k \geq 1$ and $1 \leq \ell \leq k+1$ and any number $p$ with $1 \leq p \leq \infty$, there exists a constant $C$, independent of $h$, such that

$$
\forall u \in W^{\ell, p}(\Omega), \forall T \in \mathcal{T}_{h},\left|u-R_{h}(u)\right|_{W^{1, p}(T)} \leq C h_{T}^{\ell-1}|u|_{W^{\ell, p}\left(\Delta_{T}\right)} .
$$

Remark 6. Clearly, the statement of Theorem 4.1 (resp., Theorem 4.2) extends immediately to the case where $u$ belongs to $W^{\ell, q}(\Omega)$, for any $q$ such that $W^{\ell, q}(\Omega)$ is continuously embedded into $L^{p}(\Omega)$ (resp., $W^{1, p}(\Omega)$ ). For instance, in view of (2.19), under the assumptions of Theorem 4.1, if $u$ belongs to $W^{\ell, q}(\Omega)$, we have, for all $T$ in $\mathcal{T}_{h}$

$$
\begin{aligned}
& \text { if } q \geq p,\left\|u-R_{h}(u)\right\|_{L^{p}(T)} \leq C h_{T}^{\ell} h_{T}^{2 / p-2 / q}|u|_{W^{\ell, q}\left(\Delta_{T}\right)} \\
& \text { if } q<p,\left\|u-R_{h}(u)\right\|_{L^{p}(T)} \leq C h_{T}^{\ell} \frac{1}{\rho_{T}^{2 / q-2 / p}}|u|_{W^{\ell, q}\left(\Delta_{T}\right)} .
\end{aligned}
$$

Remark 7. Combining the final remarks of sections 2 and 3 with the inequality, valid for any nonnegative real number $s$

$$
\left|\varphi_{i}\right|_{W^{s, p}(T)} \leq c h_{T}^{\frac{2}{p}-s}
$$


we derive that, for any real numbers $s$ and $t$ with $0 \leq s \leq 1$ and $s \leq t \leq k+1$ and any number $p$ with $1 \leq p \leq \infty$, the following estimate holds for any function $u$ in $W^{t, p}(\Omega)$ :

$$
\left|u-R_{h}(u)\right|_{W^{s, p}(T)} \leq C h_{T}^{t-s}\|u\|_{W^{t, p}\left(\Delta_{T}\right)} .
$$

Similarly, if $f$ is a side of an element $T$, for any real numbers $s, t$, and $p$ with $0 \leq s \leq 1$, $s+\frac{1}{p}<t \leq k+1$, and $1 \leq p<\infty$, the following estimate holds for any function $u$ in $W^{t, p}(\Omega)$ :

$$
\left|u-R_{h}(u)\right|_{W^{s, p}(f)} \leq C h_{T}^{t-s-\frac{1}{p}}\|u\|_{W^{t, p}\left(\Delta_{T}\right)} .
$$

Now we turn to the regularization of functions with imposed values on some part of the boundary. More precisely, let $\Gamma_{0}$ denote a connected subset of $\Gamma$ with positive measure. For $1 \leq p<\infty$ and $\ell \geq 1$, we want to approximate functions of the space

$$
W_{\Gamma_{0}}^{\ell, p}(\Omega)=\left\{v \in W^{\ell, p}(\Omega) ; v=0 \text { on } \Gamma_{0}\right\} .
$$

To this end, we assume that $\bar{\Omega}$ is triangulated or quadrangulated so that the end points of $\Gamma_{0}$ coincide with nodes of the triangulation. Then we number first the nodes of $\mathcal{T}_{h}$ that lie on $\Gamma_{0}$, say from 1 to $N_{0}$, and next we number the remaining nodes from $N_{0}+1$ to $N$. To ensure that the finite element functions vanish on $\Gamma_{0}$, we consider the finite element space spanned by the set of basis functions $\left\{\varphi_{i} ; N_{0}+1 \leq i \leq N\right\}$, that is,

$$
\Theta_{h, \Gamma_{0}}=\left\{\theta_{h} \in \mathcal{C}^{0}(\bar{\Omega}) ; \forall T \in \mathcal{T}_{h},\left.\theta_{h}\right|_{T} \in \mathbb{P}_{k} \text { or } \mathcal{Q}_{k}(T) \text { and } \theta_{h}=0 \text { on } \Gamma_{0}\right\} .
$$

The regularization operator $R_{h, \Gamma_{0}}$ is defined by

$$
\forall u \in W_{\Gamma_{0}}^{\ell, p}(\Omega), R_{h, \Gamma_{0}}(u)(\boldsymbol{x})=\sum_{i=N_{0}+1}^{N}\left[r_{i}(u)\right]\left(\boldsymbol{a}_{i}\right) \varphi_{i}(\boldsymbol{x}) .
$$

Clearly, $R_{h, \Gamma_{0}}$ is continuous from $W_{\Gamma_{0}}^{\ell, p}(\Omega)$ into $\Theta_{h, \Gamma_{0}}$. Let us show that it satisfies the analogues of Theorems 4.1 and 4.2 .

THEOREM 4.3. Assume that $\left(\mathcal{T}_{h}\right)_{h}$ is a regular family of triangulations or quadrangulations of $\bar{\Omega}$. For any integers $k$ and $\ell$ with $k \geq 1$ and $1 \leq \ell \leq k+1$ and any real number $p$ with $1 \leq p<\infty$, there exists a constant $C$, independent of $h$, such that

$$
\forall u \in W_{\Gamma_{0}}^{\ell, p}(\Omega), \forall T \in \mathcal{T}_{h},\left\|u-R_{h, \Gamma_{0}}(u)\right\|_{L^{p}(T)} \leq C h_{T}^{\ell}|u|_{W^{\ell, p}\left(\Delta_{T}\right)} .
$$

Proof. It suffices to consider the elements $T$ that have some nodes on $\Gamma_{0}$. Suppose again that $\Delta_{T}$ contains $n$ macroelements $\Delta_{i}$. We agree to number first, say from 1 to $n_{0}$, the nodes $\boldsymbol{a}_{i}$ that lie on $\Gamma_{0}$ and from $n_{0}+1$ to $n$ the remaining nodes. Then, owing to the support of the basis functions $\varphi_{i}$, we have

$$
\left.R_{h, \Gamma_{0}}(u)\right|_{T}=\left.\sum_{i=n_{0}+1}^{n}\left[r_{i}(u)\right]\left(\boldsymbol{a}_{i}\right) \varphi_{i}\right|_{T}=\left.R_{h}(u)\right|_{T}-\left.\sum_{i=1}^{n_{0}}\left[r_{i}(u)\right]\left(\boldsymbol{a}_{i}\right) \varphi_{i}\right|_{T} .
$$

Hence,

$$
\left\|u-R_{h, \Gamma_{0}}(u)\right\|_{L^{p}(T)} \leq\left\|u-R_{h}(u)\right\|_{L^{p}(T)}+\sum_{i=1}^{n_{0}}\left|\left[r_{i}(u)\right]\left(\boldsymbol{a}_{i}\right)\right|\left\|\varphi_{i}\right\|_{L^{p}(T)} .
$$


Next, we observe that each boundary node $\boldsymbol{a}_{i}, 1 \leq i \leq n_{0}$, belongs to a side $f$ of a triangle or quadrilateral $T^{\prime}$ contained in $\Delta_{i}\left(T^{\prime}\right.$ does not necessarily coincide with $\left.T\right)$. Thus, we have

$$
\begin{aligned}
\left|\left[r_{i}(u)\right]\left(\boldsymbol{a}_{i}\right)\right| & \leq\left\|r_{i}(u)\right\|_{L^{\infty}(f)}=\left\|\widehat{r_{i}(u)}\right\|_{L^{\infty}(\hat{f})} \\
& \leq \hat{C}_{1}\left\|\widehat{r_{i}(u)}\right\|_{L^{p}(\hat{f})}=\hat{C}_{1}\left\|\widehat{r_{i}(u)}-\hat{u}\right\|_{L^{p}(\hat{f})} \leq \hat{C}_{2}\left\|\widehat{r_{i}(u)}-\hat{u}\right\|_{W^{1, p}(\hat{T})} .
\end{aligned}
$$

Here we have used first the fact that $\widehat{r_{i}(u)}$ belongs to a finite-dimensional space on $\hat{T}$, next the fact that $\hat{u}$ vanishes on $\hat{f}$, and finally the trace theorem on $\hat{T}$. Therefore, if $T^{\prime}$ is a triangle,

$$
\left|\left[r_{i}(u)\right]\left(\boldsymbol{a}_{i}\right)\right| \leq \hat{C}_{3}\left|\operatorname{det}\left(B_{T^{\prime}}\right)\right|^{-1 / p}\left(\left\|r_{i}(u)-u\right\|_{L^{p}\left(T^{\prime}\right)}+\left\|B_{T^{\prime}}\right\|\left|r_{i}(u)-u\right|_{W^{1, p}\left(T^{\prime}\right)}\right),
$$

or if $T^{\prime}$ is a quadrilateral,

$$
\begin{aligned}
\left|\left[r_{i}(u)\right]\left(\boldsymbol{a}_{i}\right)\right| \leq & \hat{C}_{4}\left|J_{T^{\prime}}^{-1}\right|_{L^{\infty}\left(T^{\prime}\right)}^{1 / p}\left(\left\|r_{i}(u)-u\right\|_{L^{p}\left(T^{\prime}\right)}\right. \\
& \left.+\left\|D \mathcal{F}_{T^{\prime}}\right\|_{L^{\infty}(\hat{T})}\left|r_{i}(u)-u\right|_{W^{1, p}\left(T^{\prime}\right)}\right) .
\end{aligned}
$$

Then (4.12) follows readily from (4.14) or (4.15) combined with (4.4), the fact that $T$ and $T^{\prime}$ belong to the same macroelement $\Delta_{i}$, and Theorems 2.1 and 2.2 or 3.4 and 3.5 .

Since the gist of the above proof consists in deriving an upper bound for $\left|\left[r_{i}(u)\right]\left(\boldsymbol{a}_{i}\right)\right|$, it is clear that this proof can be easily adapted to establish the next result.

THEOREM 4.4. Assume that $\left(\mathcal{T}_{h}\right)_{h}$ is a regular family of triangulations or quadrangulations of $\bar{\Omega}$. For any integers $k$ and $\ell$ with $k \geq 1$ and $1 \leq \ell \leq k+1$ and any real number $p$ with $1 \leq p<\infty$, there exists a constant $C$, independent of $h$, such that

$$
\forall u \in W_{\Gamma_{0}}^{\ell, p}(\Omega), \forall T \in \mathcal{T}_{h},\left\|u-R_{h, \Gamma_{0}}(u)\right\|_{W^{1, p}(T)} \leq C h_{T}^{\ell-1}|u|_{W^{\ell, p}\left(\Delta_{T}\right)} .
$$

Remark 8. Estimates (4.8) to (4.10) still hold with the operator $R_{h}$ replaced by $R_{h, \Gamma_{0}}$.

Thus, we have exhibited two regularization operators, the second one being designed for handling functions that vanish on part of the boundary. They have optimal approximation properties in a large number of Sobolev norms, and the optimality concerns both the order of the approximation and its local behavior (the ratio of the diameter of $\Delta_{T}$ to the diameter of $T$ is bounded independently of $h$ ).

5. Applications to a lifting operator and residual error indicators. A regularizing operator is a very useful theoretical tool. Among its best-known applications is the proof of the "inf-sup" condition that must be satisfied by spaces that discretize the Stokes or Navier-Stokes problem; cf. [11, Chapter II], for instance. But it is far from being its only application, and to illustrate this point, we have chosen to describe, on one hand, the construction of a discrete lifting operator that was suggested by $\mathrm{O}$. Widlund [17] and, on the other hand, the derivation of optimal estimates for a family of residual indicators.

Construction of a lifting operator. Again let $\Gamma_{0}$ denote a subset of $\Gamma$ with positive measure, and assume that $\bar{\Omega}$ is triangulated, by a regular family of triangulations (or quadrangulations) $\left(\mathcal{T}_{h}\right)_{h}$, in such a way that the end points of $\Gamma_{0}$ coincide with nodes of the triangulation. Here also, we number first the nodes of $\mathcal{T}_{h}$ that lie on $\Gamma_{0}$, say 
from 1 to $N_{0}$, and next we number the remaining nodes from $N_{0}+1$ to $N$. Then we associate with $\mathcal{T}_{h}$ the finite element space $\Theta_{h}$, defined by (2.1) or (3.3) for some integer $k \geq 1$, and we denote by $W_{h}$ the space of traces on $\Gamma_{0}$ of all functions of $\Theta_{h}$. For $1 \leq p<\infty$, we wish to construct an operator $L_{h}$ from $W_{h}$ into $\Theta_{h}$ that lifts the trace (for all $w_{h}$ in $W_{h}$, the trace of $L_{h}\left(w_{h}\right)$ on $\Gamma_{0}$ coincides with $w_{h}$ ) and that is continuous with a norm independent of $h$. To this end, we introduce first a standard lifting operator $L$ that is continuous from $W^{1-1 / p, p}\left(\Gamma_{0}\right)$ into $W^{1, p}(\Omega)$. Next, we regularize $L\left(w_{h}\right)$ by the operator $R_{h}$ defined by (4.1). Finally, since the values of $R_{h}\left(L\left(w_{h}\right)\right)$ do not necessarily coincide with those of $w_{h}$ on $\Gamma_{0}$, we correct them by the technique of the preceding section; thus we set

$$
L_{h}\left(w_{h}\right)(\boldsymbol{x})=\sum_{i=1}^{N_{0}} w_{h}\left(\boldsymbol{a}_{i}\right) \varphi_{i}(\boldsymbol{x})+\sum_{i=N_{0}+1}^{N}\left[R_{h}\left(L\left(w_{h}\right)\right)\right]\left(\boldsymbol{a}_{i}\right) \varphi_{i}(\boldsymbol{x}) .
$$

Obviously, the trace of $L_{h}\left(w_{h}\right)$ on $\Gamma_{0}$ coincides with $w_{h}$. The next theorem establishes the uniform stability of $L_{h}$.

THEOREM 5.1. Assume that $\left(\mathcal{T}_{h}\right)_{h}$ is a regular family of triangulations or quadrangulations of $\bar{\Omega}$, and let $L_{h}$ be defined by (5.1). For any integer $k \geq 1$ and any real number $p$ with $1 \leq p<\infty$, there exists a constant $C$, independent of $h$, such that

$$
\forall w_{h} \in W_{h},\left\|L_{h}\left(w_{h}\right)\right\|_{W^{1, p}(\Omega)} \leq C\left\|w_{h}\right\|_{W^{1-1 / p, p}\left(\Gamma_{0}\right)} .
$$

Proof. For any $w_{h}$ in $W_{h}$, we write

$$
\left\|L_{h}\left(w_{h}\right)\right\|_{W^{1, p}(\Omega)} \leq\left\|R_{h}\left(L\left(w_{h}\right)\right)\right\|_{W^{1, p}(\Omega)}+\left\|R_{h}\left(L\left(w_{h}\right)\right)-L_{h}\left(w_{h}\right)\right\|_{W^{1, p}(\Omega)} .
$$

The first term is estimated by Theorems 4.1 and 4.2 with $\ell=1$ and by the standard property of the lifting operator $L$,

$$
\left\|R_{h}\left(L\left(w_{h}\right)\right)\right\|_{W^{1, p}(\Omega)} \leq C_{1}\left\|L\left(w_{h}\right)\right\|_{W^{1, p}(\Omega)} \leq C_{2}\left\|w_{h}\right\|_{W^{1-1 / p, p}\left(\Gamma_{0}\right)} .
$$

By construction, the second term has the expression

$$
R_{h}\left(L\left(w_{h}\right)\right)-L_{h}\left(w_{h}\right)=\sum_{i=1}^{N_{0}}\left[R_{h}\left(L\left(w_{h}\right)\right)-w_{h}\right]\left(\boldsymbol{a}_{i}\right) \varphi_{i} .
$$

Then we proceed as in the preceding section. Let $T$ be an element of $\mathcal{T}_{h}$ such that some nodes of $T$ lie on $\Gamma_{0}$. Suppose again that $\Delta_{T}$ contains $n$ macroelements $\Delta_{i}$. We agree to number first, say from 1 to $n_{0}$, the nodes $\boldsymbol{a}_{i}$ that lie on $\Gamma_{0}$ and from $n_{0}+1$ to $n$ the remaining nodes. Then, owing to the support of the basis functions $\varphi_{i}$, we have

$$
\left\|R_{h}\left(L\left(w_{h}\right)\right)-L_{h}\left(w_{h}\right)\right\|_{W^{1, p}(T)} \leq \sum_{i=1}^{n_{0}}\left|\left[R_{h}\left(L\left(w_{h}\right)\right)-w_{h}\right]\left(\boldsymbol{a}_{i}\right)\right|\left\|\varphi_{i}\right\|_{W^{1, p}(T)} .
$$

On one hand, if $T$ is a triangle,

$$
\left\|\varphi_{i}\right\|_{W^{1, p}(T)} \leq\left|\operatorname{det}\left(B_{T}\right)\right|^{1 / p}\left(\left\|\hat{\varphi}_{i}\right\|_{L^{p}(\hat{T})}^{p}+\left\|B_{T}^{-1}\right\|^{p}\left|\hat{\varphi}_{i}\right|_{W^{1, p}(\hat{T})}^{p}\right)^{1 / p},
$$

and since the leading term is the one with the factor $\left\|B_{T}^{-1}\right\|$, we can write

$$
\left\|\varphi_{i}\right\|_{W^{1, p}(T)} \leq \hat{C}_{1}\left|\operatorname{det}\left(B_{T}\right)\right|^{1 / p}\left\|B_{T}^{-1}\right\| .
$$


Similarly, if $T$ is a quadrilateral, we have

$$
\left\|\varphi_{i}\right\|_{W^{1, p}(T)} \leq \hat{C}_{2}\left\|J_{T}\right\|_{L^{\infty}(\hat{T})}^{1 / p}\left\|D \mathcal{F}_{T}^{-1}\right\|_{L^{\infty}(T)} .
$$

On the other hand, to simplify the discussion, we assume that $\boldsymbol{a}_{i}$ belongs to a side $f$ of $T$ that lies on $\Gamma_{0}$. Then

$$
\begin{aligned}
\mid\left[R_{h}\left(L\left(w_{h}\right)\right)-w_{h}\right] & \left.\left(\boldsymbol{a}_{i}\right) \mid \leq\left\|R_{h}\left(L\left(w_{h}\right)\right)-w_{h}\right\|_{L^{\infty}(f)}=\| R_{h} \widehat{\left(L\left(w_{h}\right)\right.}\right)-\hat{w}_{h} \|_{L^{\infty}(\hat{f})} \\
& \left.\left.\leq \hat{C}_{3} \| R_{h} \widehat{\left(L\left(w_{h}\right)\right.}\right)-\hat{w}_{h}\left\|_{L^{p}(\hat{f})} \leq \hat{C}_{4}\right\| R_{h} \widehat{\left(L\left(w_{h}\right)\right.}\right)-\widehat{L\left(w_{h}\right)} \|_{W^{1, p}(\hat{T})},
\end{aligned}
$$

where in the last inequality, we have used the fact that

$$
\widehat{L\left(w_{h}\right)}=\hat{w}_{h} \text { on } \hat{f}
$$

in order to apply the trace theorem on $\hat{T}$. Therefore, if $T$ is a triangle,

$$
\begin{array}{r}
\left|\left[R_{h}\left(L\left(w_{h}\right)\right)-w_{h}\right]\left(\boldsymbol{a}_{i}\right)\right| \leq \hat{C}_{5}\left|\operatorname{det}\left(B_{T}\right)\right|^{-1 / p}\left(\left\|R_{h}\left(L\left(w_{h}\right)\right)-L\left(w_{h}\right)\right\|_{L^{p}(T)}^{p}\right. \\
\left.+\left\|B_{T}\right\|^{p}\left|R_{h}\left(L\left(w_{h}\right)\right)-L\left(w_{h}\right)\right|_{W^{1, p}(T)}^{p}\right)^{1 / p},
\end{array}
$$

and if $T$ is a quadrilateral,

$$
\begin{gathered}
\left|\left[R_{h}\left(L\left(w_{h}\right)\right)-w_{h}\right]\left(\boldsymbol{a}_{i}\right)\right| \leq \hat{C}_{6}\left\|J_{T}^{-1}\right\|_{L^{\infty}(T)}^{1 / p}\left(\left\|R_{h}\left(L\left(w_{h}\right)\right)-L\left(w_{h}\right)\right\|_{L^{p}(T)}^{p}\right. \\
\left.+\left\|D \mathcal{F}_{T}\right\|_{L^{\infty}(\hat{T})}^{p}\left|R_{h}\left(L\left(w_{h}\right)\right)-L\left(w_{h}\right)\right|_{W^{1, p}(T)}^{p}\right)^{1 / p} .
\end{gathered}
$$

Then (5.2) follows from (5.3), (5.4), and (5.7) combined with (5.5) if $T$ is a triangle, or (5.8) combined with (5.6) if $T$ is a quadrilateral, together with Theorems 4.1 and 4.2 .

Residual indicators on a quadrilateral mesh. The residual indicators for the Poisson equation are known to satisfy optimal estimates when associated with a standard conforming discretization on a triangular mesh (or tetrahedral mesh in three dimensions); see [16] or [3]. The aim of this section is to extend these results to the case of any mesh made of convex quadrilaterals.

So, assuming that the data $g$ belong to $L^{2}(\Omega)$, we consider the Poisson equation

$$
\begin{cases}-\Delta u=g & \text { in } \Omega, \\ u=0 & \text { on } \partial \Omega .\end{cases}
$$

Since the domain $\Omega$ has a polygonal boundary, we introduce a regular family $\left(\mathcal{T}_{h}\right)_{h}$ of quadrangulations of $\bar{\Omega}$, and, for the discrete space $\Theta_{h}$ defined in (3.3), we set

$$
\Theta_{h}^{0}=\Theta_{h} \cap H_{0}^{1}(\Omega) .
$$

Then, the discrete problem reads

Find $u_{h}$ in $\Theta_{h}^{0}$ such that

$$
\forall v_{h} \in \Theta_{h}^{0}, \quad \int_{\Omega} \operatorname{grad} u_{h} \cdot \operatorname{grad} v_{h} d \mathbf{x}=\int_{\Omega} g(\mathbf{x}) v_{h}(\mathbf{x}) d \mathbf{x} .
$$

The standard a priori estimate is

$$
\left|u-u_{h}\right|_{H^{1}(\Omega)} \leq c h^{\ell-1}|u|_{H^{\ell}(\Omega)}
$$


when the solution $u$ is supposed to belong to $H^{\ell}(\Omega), 1 \leq \ell \leq k+1$.

Next, for a nonnegative integer $m$, we introduce the finite element space

$$
\Lambda_{h}=\left\{\lambda_{h} \in L^{2}(\Omega) ; \forall T \in \mathcal{T}_{h}, \lambda_{h \mid T} \in \mathcal{Q}_{m}(T)\right\},
$$

and we choose an approximation $g_{h}$ of the data $g$ in $\Lambda_{h}$. Also, with each quadrilateral $T$ of $\mathcal{T}_{h}$, we associate the set $\mathcal{E}_{T}$ of sides of $T$ which are not contained in the boundary of $\Omega$ and we denote by $h_{f}$ the length of each $f$ in $\mathcal{E}_{T}$.

We are now in a position to define the family of indicators $\left(\eta_{T}\right)_{T \in \mathcal{T}_{h}}$ :

$$
\eta_{T}=h_{T}\left\|g_{h}+\Delta u_{h}\right\|_{L^{2}(T)}+\frac{1}{2} \sum_{f \in \mathcal{E}_{T}} h_{f}^{\frac{1}{2}}\left\|\left[\frac{\partial u_{h}}{\partial n}\right]\right\|_{L^{2}(f)},
$$

where $\left[\frac{\partial u_{h}}{\partial n}\right]$ denotes the jump of $\frac{\partial u_{h}}{\partial n}$ across $f$. The following two theorems sum up the optimal properties of these indicators.

THEOREM 5.2. The family of indicators defined in (5.12) satisfies

$$
\left|u-u_{h}\right|_{H^{1}(\Omega)} \leq c\left(\sum_{T \in \mathcal{T}_{h}}\left(\eta_{T}^{2}+h_{T}^{2}\left\|g-g_{h}\right\|_{L^{2}(T)}^{2}\right)\right)^{\frac{1}{2}} .
$$

Proof. It relies on the formula

$$
\left|u-u_{h}\right|_{H^{1}(\Omega)}=\sup _{w \in H_{0}^{1}(\Omega)} \frac{\int_{\Omega} \operatorname{grad}\left(u-u_{h}\right) \cdot \operatorname{grad} w d \mathbf{x}}{|w|_{H^{1}(\Omega)}} .
$$

It follows from (5.11) that, for any $w_{h}$ in $\Theta_{h}^{0}$,

$$
\begin{aligned}
\int_{\Omega} \operatorname{grad}\left(u-u_{h}\right) \cdot \operatorname{grad} w d \mathbf{x} & =\int_{\Omega} \operatorname{grad}\left(u-u_{h}\right) \cdot \operatorname{grad}\left(w-w_{h}\right) d \mathbf{x} \\
& =\sum_{T \in \mathcal{T}_{h}} \int_{T} \operatorname{grad}\left(u-u_{h}\right) \cdot \operatorname{grad}\left(w-w_{h}\right) d \mathbf{x},
\end{aligned}
$$

so that integrating by parts and using a Cauchy-Schwarz inequality leads to

$$
\begin{aligned}
& \int_{\Omega} \operatorname{grad}\left(u-u_{h}\right) \cdot \operatorname{grad} w d \mathbf{x} \\
& \leq \sum_{T \in \mathcal{T}_{h}}\left(\left\|g+\Delta u_{h}\right\|_{L^{2}(T)}\left\|w-w_{h}\right\|_{L^{2}(T)}+\frac{1}{2} \sum_{f \in \mathcal{E}_{T}}\left\|\left[\frac{\partial u_{h}}{\partial n}\right]\right\|_{L^{2}(f)}\left\|w-w_{h}\right\|_{L^{2}(f)}\right) .
\end{aligned}
$$

Now, we take $w_{h}=R_{h}^{0} w$, where $R_{h}^{0}$ denotes the regularization operator $R_{h, \Gamma_{0}}$ for $\Gamma_{0}=\partial \Omega$, and we derive from (4.16) and the analogue of (4.10) that

$$
\begin{aligned}
& \int_{\Omega} \operatorname{grad}\left(u-u_{h}\right) \cdot \operatorname{grad} w d \mathbf{x} \\
& \leq c \sum_{T \in \mathcal{T}_{h}}\left(h_{T}\left\|g+\Delta u_{h}\right\|_{L^{2}(T)}+\frac{1}{2} \sum_{f \in \mathcal{E}_{T}} h_{f}^{\frac{1}{2}}\left\|\left[\frac{\partial u_{h}}{\partial n}\right]\right\|_{L^{2}(f)}\right)|w|_{H^{1}\left(\Delta_{T}\right)} .
\end{aligned}
$$

Using once more a Cauchy-Schwarz inequality and also inserting $g_{h}$ with a triangular inequality, we obtain (5.13). 
The arguments for deriving an upper bound for the $\eta_{T}$ are strictly the same as in the case of a triangular mesh, so we refer to Verfürth [15], [16], [3] for the proof of the following theorem.

THEOREM 5.3. The family of indicators defined in (5.12) satisfies, for all $T$ in $\mathcal{T}_{h}$,

$$
\eta_{T} \leq c\left(\left|u-u_{h}\right|_{H^{1}\left(\Omega_{T}\right)}+\left\|g-g_{h}\right\|_{L^{2}\left(\Omega_{T}\right)}\right),
$$

where $\Omega_{T}$ denotes the union of all quadrilaterals in $\mathcal{T}_{h}$ which share at least a side with $T$.

The main consequence of Theorem 5.2 is that a bound for the error can be computed explicitly, up to a multiplicative constant, without any further assumption on the regularity of the exact solution. However, the constant is not easy to evaluate; we refer to Babuška, Durán, and Rodríguez [1] for interesting tentatives in this direction. And, by combining the two theorems, each indicator $\eta_{T}$ appears to be fairly representative of the local error, thus leading to an efficient refinement of the mesh. Complete results are given by Verfürth (see [16] and the references therein).

\section{REFERENCES}

[1] I. Babuška, R. Durán, And R. Rodríguez, Analysis of the efficiency of an a posteriori error estimator for linear triangular finite elements, SIAM J. Numer. Anal., 29 (1992), pp. 947-964.

[2] C. Bernardi, Optimal finite element interpolation on curved domains, SIAM J. Numer. Anal., 26 (1989), pp. 1212-1240.

[3] C. Bernardi, B. Métivet, and R. Verfürth, Analyse numérique d'indicateurs d'erreur, Technical report, Laboratoire d'Analyse Numérique de l'Université Pierre et Marie Curie, Paris, 1993.

[4] S. Brenner And R. Scott, The Mathematical Theory of Finite Element Methods, Texts Appl. Math. 15, Springer-Verlag, New York, 1994.

[5] P.G. Ciarlet, Basic Error Estimates for Elliptic Problems, in Handbook of Numerical Analysis, Vol. II, P.G. Ciarlet and J.-L. Lions, eds., North-Holland, Amsterdam, 1991.

[6] P.G. Ciarlet AND P.-A. RAViaRT, The combined effect of curved boundaries and numerical integration in isoparametric finite element methods, in The Mathematical Foundations of the Finite Element Method with Application to Partial Differential Equations, A.K. Aziz, ed., Academic Press, New York, 1972, pp. 409-474.

[7] P. ClÉment, Approximation by finite element functions using local regularization, RAIRO Anal. Numér., 9 (1975), pp. 77-84.

[8] J. Deny and J.-L. Lions, Les espaces du type de Beppo Levi, Annales de l'Institut Fourier (Grenoble), 5 (1953-54), pp. 305-370.

[9] T. Dupont and R. Scott, Polynomial approximation of functions in Sobolev spaces, Math. Comp., 34 (1980), pp. 441-463.

[10] R.G. DuRÁN, On polynomial approximation in Sobolev spaces, SIAM J. Numer. Anal., 20 (1983), pp. 985-988.

[11] V. Girault and P.-A. Raviart, Finite Element Methods for Navier-Stokes Equations: Theory and Algorithms, Springer Ser. Comput. Math. 5, Springer-Verlag, New York, 1986.

[12] J.-L. Lions and E. Magenes, Problèmes aux limites non homogènes et applications, Dunod, Paris, 1970.

[13] J. NeČas, Les méthodes directes en théorie des équations elliptiques, Masson, Paris, 1967.

[14] L.R. SCOTT AND S. ZhANG, Finite element interpolation of nonsmooth functions satisfying boundary conditions, Math. Comp., 54 (1990), pp. 483-493.

[15] R. VerfürTh, A posteriori error estimators for the Stokes equations, Numer. Math., 55 (1989), pp. 309-325.

[16] R. Verfürth, A Review of A Posteriori Error Estimation and Adaptive Mesh-Refinement Techniques, Wiley \& Teubner, Chichester and Stuttgart, 1996.

[17] O.B. WidLund, An extension theorem for finite element spaces with three applications, in Numerical Techniques in Continuum Mechanics, Proc. Second GAMM Seminar, Kiel, 1986. 